TIFR/TH/07-36

\title{
Collective three-flavor oscillations of supernova neutrinos
}

\author{
Basudeb Dasgupta and Amol Dighe \\ Tata Institute of Fundamental Research, Homi Bhabha Road, Mumbai 400005, India
}

Neutrinos and antineutrinos emitted from a core collapse supernova interact among themselves, giving rise to collective flavor conversion effects that are significant near the neutrinosphere. We develop a formalism to analyze these collective effects in the complete three-flavor framework. It naturally generalizes the spin-precession analogy to three flavors and is capable of analytically describing phenomena like vacuum/MSW oscillations, synchronized oscillations, bipolar oscillations and spectral split. Using the formalism, we demonstrate that the flavor conversions may be "factorized" into two-flavor oscillations with hierarchical frequencies. We explicitly show how the three-flavor solution may be constructed by combining two-flavor solutions. For a typical supernova density profile, we identify an approximate separation of regions where distinctly different flavor conversion mechanisms operate, and demonstrate the interplay between collective and MSW effects. We pictorialize our results in terms of the " $\mathbf{e}_{3}-\mathbf{e}_{8}$ triangle" diagram, which is a tool that can be used to visualize three-neutrino flavor conversions in general, and offers insights into the analysis of the collective effects in particular.

PACS numbers: 14.60.Pq, 97.60.Bw

\section{INTRODUCTION}

Neutrinos emitted from a core collapse supernova carry information about the primary fluxes, neutrino masses and mixing, and SN dynamics [1, 2]. Neutrinos, produced in the region of the neutrinosphere, freestream outwards and pass through the core, mantle and envelope of the star. The drastically different environments in these regions, consisting of varying densities of ordinary matter, neutrinos and antineutrinos, affect flavor conversions among neutrinos. Multiple shock fronts and turbulence generated during the explosion may also affect the neutrino flavor conversions.

The traditional picture of flavor conversions in a $\mathrm{SN}$ is based on the assumption that the effect of neutrino-neutrino interactions is small. In this picture, neutrinos that are produced approximately as mass eigenstates at very high ambient matter density in the core propagate outwards from the neutrinosphere. Flavor conversion proceeds most efficiently at the electron density corresponding to the MSW resonance [3, 4]. The outcoming incoherent mixture of vacuum mass eigenstates is observed at a detector to be a combination of primary fluxes of the three neutrino flavors. 
This scenario of resonant neutrino conversions in a SN [5] has been studied extensively to probe neutrino mixings and SN dynamics. The work has focussed on the determination of mass hierarchy and signatures of a non-zero $\theta_{13}[6,7]$, earth matter effects on the neutrino fluxes when they pass through matter [8 10], shock wave effects on observable neutrino spectra and their model independent signatures [11 16]. Recently, possible interference effects for multiple resonances [17], the role of turbulence in washing out shock wave effects [18 20], and time variation of the signal [21] have also been explored.

However, neutrino and antineutrino densities near the neutrinosphere are extremely high $\left(10^{30-35}\right.$ per $\left.\mathrm{cm}^{3}\right)$, which make the neutrino-neutrino interactions significant. Such a dense gas of neutrinos and antineutrinos is coupled to itself, making its evolution nonlinear. The flavor offdiagonal terms can be sizeable, and significant flavor conversion is possible [22, 23]. A formalism to study flavor evolution of such dense relativistic neutrino gases was developed in [24 26], where a set of quantum kinetic equations for their evolution were written down. These equations have been studied in detail, though mostly in the two-flavor approximation, and the nature of flavor evolution has been identified [27 30]. It was eventually understood that a dense gas of neutrinos displays collective flavor conversion, i.e. neutrinos of all energies oscillate together, through synchronized oscillations [31] and/or bipolar oscillations [32, 33]. Another remarkable effect of these interactions is a partial or complete swapping of the energy spectra of two neutrino flavors, called step-wise spectral swapping or simply spectral splits, as the neutrinos transit from a region where collective effects dominate to a region where neutrino density is low [34, 35].

The nonlinear effects in the context of SNe were considered in [36-40]. Recent two-flavor simulations showed that the collective effects affect neutrino flavor conversions substantially [41, 42]. Different collective flavor transformations seem to play a part in different regions of the star. Many features of the results of these simulations can be understood from the "single-angle" approximation, i.e. ignoring the dependence of the initial launching angle of neutrinos on the evolutions of neutrino trajectories [32 35, 43 48]. Angular dependence of flavor evolution can give rise to additional angle dependent features observed in two-flavor simulations [41, 42], or to decoherence effects [29, 49]. For a realistic asymmetry between neutrino and antineutrino fluxes, such angle dependent effects are likely to be small [47, 48]. Recently collective effects have also been numerically investigated in the context of the neutronization-burst phase of $\mathrm{O}-\mathrm{Ne}-\mathrm{Mg}$ supernovae [50]. The impact of these nonlinear interactions has also been studied in the context of cosmological neutrino flavor equilibration in the early Universe where the synchronized oscillations play a significant part [51 59$]$. 
Most of the analytical results in this area are in the two-flavor approximation, where the equations describing flavor transformations are similar to the equations of motion of a gyroscope. As a result, two-flavor results are now fairly well understood analytically, with the exception of possibly two issues, viz. decoherence (or lack of it) for asymmetric systems [47], and the existence of the antineutrino spectral split [48]. In this work we focus on the effects of the mixing of the third flavor. We present an analytical framework to study three-flavor effects and demonstrate an approximate factorization of the full three-flavor problem into simpler two-flavor problems, when $\Delta m_{\odot}^{2} \ll\left|\Delta m_{\text {atm }}^{2}\right|$ and $\theta_{13} \ll 1$. We also develop the "e $\mathbf{e}_{3}-\mathbf{e}_{8}$ triangle" diagram, a tool that can be used to visualize the three neutrino flavor conversions in general, and allows one to gauge the extent of additional effects of the third flavor. We numerically study collective flavor transformations for a typical SN density profile, identify regions where different flavor conversion mechanisms operate, and explain the features of the spectra using our formalism.

The outline of this paper is as follows. In Sec. III we review the equations of motion of a dense gas of neutrinos in steady state. We specialize these equations to spherical geometry in the single-angle half-isotropic approximation, and write the three-flavor analogue of the gyroscope equations, by introducing the eight-dimensional Bloch vectors. We recover the two-flavor limit of those equations, and recognize an approximate factorization of the three-flavor problem (reminiscent of the $H-L$ factorization in the standard picture) into smaller two-flavor problems. We show that survival probabilities can be written down in a simple form, purely in terms of the solutions to the two-flavor problems, as long as the frequencies governing the oscillations are hierarchically separated. In Sec. III, we illustrate the above factorization for vacuum/MSW oscillations as well as collective synchronized oscillations. We also explain the three-flavor features of bipolar oscillations and spectral splits qualitatively and pictorially. In Sec. IV, we calculate the flavor conversion probabilities numerically for a typical SN matter profile, and identify the additional features due to the third neutrino. We conclude in Sec. $\mathrm{V}$ with a summary of our results and comments about directions of future investigation.

\section{THREE-FLAVOR FORMALISM}

In this section we derive the steady state equations of motion for an ensemble of dense gas of three-flavor neutrinos, as a straightforward generalization of the corresponding equations in the two-flavor case. 


\section{A. Hamiltonian and Equations of Motion}

We denote a neutrino of momentum $\mathbf{p}$ at time $t$ at position $\mathbf{r}$ by $\nu(\mathbf{p}, \mathbf{r}, t)$, and work in the modified flavor basis $\left(\nu_{e}, \nu_{x}, \nu_{y}\right)$ defined such that $\left(\nu_{e} \nu_{x} \nu_{y}\right)^{T}=R_{23}^{\dagger}\left(\theta_{23}\right)\left(\nu_{e} \nu_{\mu} \nu_{\tau}\right)^{T}$, where $R_{23}$ is the rotation matrix and $\theta_{23}$ the neutrino mixing angle in the 2-3 plane. ${ }^{1}$ In this basis, the density matrix for $n_{\nu}(\mathbf{p}, \mathbf{r}, t)$ neutrinos with momenta between $\mathbf{p}$ and $\mathbf{p}+d \mathbf{p}$ at any position between $\mathbf{r}$ and $\mathbf{r}+d \mathbf{r}$ may be written as

$$
\rho_{\nu_{\alpha} \nu_{\beta}}(\mathbf{p}, \mathbf{r}, t) \equiv \frac{1}{n_{\nu}(\mathbf{p}, \mathbf{r}, t)} \sum|\nu(\mathbf{p}, \mathbf{r}, t)\rangle\left\langle\left.\nu(\mathbf{p}, \mathbf{r}, t)\right|_{\alpha \beta},\right.
$$

where $\alpha, \beta=e, x, y$ and the summation is over all $n_{\nu}(\mathbf{p}, \mathbf{r}, t)$ neutrinos. Note that the density matrix is normalized to have unit trace, but the neutrino density itself is $n_{\nu}(\mathbf{p}, \mathbf{r}, t)$, which typically falls off as $1 / r^{2}$ from the source. The number density of neutrinos with flavor $\alpha$ is obtained through

$$
n_{\nu_{\alpha}}(\mathbf{p}, \mathbf{r}, t)=n_{\nu}(\mathbf{p}, \mathbf{r}, t) \rho_{\nu_{\alpha} \nu_{\alpha}}(\mathbf{p}, \mathbf{r}, t)
$$

We define the analogous quantities for antineutrinos similarly, but with a reversed order of flavor indices to keep the form of equations of motion identical [25], and denote them with a "bar" over the corresponding variables for neutrinos.

The effective Hamiltonian in the modified flavor basis for neutrinos $\nu(\mathbf{p}, \mathbf{r}, t)$ of energy $E \approx p=$ $|\mathbf{p}|$ in vacuum is

$$
H_{v a c}(p)=U M^{2} U^{\dagger} / 2 p
$$

where the masses and the mixing matrix are parametrized as

$$
\begin{aligned}
M & \equiv \operatorname{Diag}\left(m_{1}, m_{2}, m_{3}\right) \\
U & \equiv R_{23}^{\dagger}\left(\theta_{23}\right) R_{23}\left(\theta_{23}\right) R_{13}\left(\theta_{13}\right) R_{12}\left(\theta_{12}\right),
\end{aligned}
$$

with $R_{i j}$ being the rotation matrices in the $i-j$ plane. In this work, we take the value of the $C P$-violating phase in neutrino sector to be zero. Now $H_{v a c}(p)$ may be explicitly written as

$$
\begin{aligned}
H_{v a c}(p) & =\frac{\Delta m_{13}^{2}}{2 p}\left(\begin{array}{ccc}
s_{13}^{2} & 0 & c_{13} s_{13} \\
0 & 0 & 0 \\
c_{13} s_{13} & 0 & c_{13}^{2}
\end{array}\right) \\
& +\frac{\Delta m_{12}^{2}}{2 p}\left(\begin{array}{ccc}
c_{13}^{2} s_{12}^{2} & c_{12} c_{13} s_{12} & -c_{13} s_{12}^{2} s_{13} \\
c_{12} c_{13} s_{12} & c_{12}^{2} & -c_{12} s_{12} s_{13} \\
-c_{13} s_{12}^{2} s_{13} & -c_{12} s_{12} s_{13} & s_{12}^{2} s_{13}^{2}
\end{array}\right),
\end{aligned}
$$

\footnotetext{
${ }^{1}$ This basis has also been denoted in the literature as $\left(\nu_{e}, \nu_{\mu^{\prime}}, \nu_{\tau^{\prime}}\right)[6]$.
} 
where $\Delta m_{i j}^{2}=m_{j}^{2}-m_{i}^{2}$ and other symbols have their usual meanings. In matter, neutrinos feel the Mikheyev-Smirnov-Wolfenstein (MSW) potential [3, 4] due to charged leptons ${ }^{2}$

$$
V(\mathbf{r}, t)=\sqrt{2} G_{F} n_{e^{-}}(\mathbf{r}, t) \operatorname{Diag}(1,0,0)
$$

that adds to the Hamiltonian, where $n_{e^{-}}(\mathbf{r})$ is the net electron number density at $\mathbf{r}$. The effective Hamiltonian also includes the effects of neutrino-neutrino interactions, which to the leading order in $G_{F}$ depend only on forward scattering and contribute [24-26]

$$
H_{\nu \nu}(\mathbf{p}, \mathbf{r}, t)=\sqrt{2} G_{F} \int \frac{d^{3} \mathbf{q}}{(2 \pi)^{3}} \kappa_{\mathbf{p q}}\left(n_{\nu}(\mathbf{q}, \mathbf{r}, t) \rho(\mathbf{q}, \mathbf{r}, t)-\bar{n}_{\nu}(\mathbf{q}, \mathbf{r}, t) \bar{\rho}(\mathbf{q}, \mathbf{r}, t)\right) .
$$

The interaction strength is dependent on the angular separation of the momenta of the interacting particles, and is given by $\kappa_{\mathbf{p q}} \equiv 1-\cos \theta_{\mathbf{p q}}$, where $\theta_{\mathbf{p q}}$ is the angle between $\mathbf{p}$ and $\mathbf{q}$.

The equation of motion for the density matrix is

$$
\frac{d}{d t} \rho(\mathbf{p}, \mathbf{r}, t)=-i[H(\mathbf{p}, \mathbf{r}, t), \rho(\mathbf{p}, \mathbf{r}, t)]+\frac{\partial}{\partial t} \rho(\mathbf{p}, \mathbf{r}, t)
$$

In the steady state (no explicit time dependence in the Hamiltonian and initial conditions) and ignoring external forces (terms depending on $d \mathbf{p} / d t$ ), we can drop the time dependence in the problem. Writing $\mathbf{v}=d \mathbf{r} / d t$ we have the equations of motion for $\rho(\mathbf{p}, \mathbf{r})$ and $\bar{\rho}(\mathbf{p}, \mathbf{r})$ as [61]

$$
\begin{aligned}
& \mathbf{v} \cdot \partial_{\mathbf{r}} \rho(\mathbf{p}, \mathbf{r})=-i\left[+H_{v a c}(p)+V(\mathbf{r})+H_{\nu \nu}(\mathbf{p}, \mathbf{r}), \rho(\mathbf{p}, \mathbf{r})\right] \\
& \mathbf{v} \cdot \partial_{\mathbf{r}} \bar{\rho}(\mathbf{p}, \mathbf{r})=-i\left[-H_{v a c}(p)+V(\mathbf{r})+H_{\nu \nu}(\mathbf{p}, \mathbf{r}), \bar{\rho}(\mathbf{p}, \mathbf{r})\right] .
\end{aligned}
$$

The effect of ordinary matter can be "rotated away" by working in the interaction picture [41, 44]. We employ an operator $O(\mathbf{r})$ under which a matrix $A$ transforms to

$$
A^{\text {int }}(\mathbf{r})=O(\mathbf{r}) A O^{-1}(\mathbf{r})
$$

where

$$
O(\mathbf{r})=\exp \left(i \int_{0}^{\mathbf{r}} d \mathbf{r}^{\prime} V\left(\mathbf{r}^{\prime}\right)\right)
$$

This choice simplifies the equations of motion by removing the matter term, giving us

$$
\begin{aligned}
& \mathbf{v} \cdot \partial_{\mathbf{r}} \rho^{i n t}(\mathbf{p}, \mathbf{r})=-i\left[+H_{v a c}^{i n t}(p, \mathbf{r})+H_{\nu \nu}^{i n t}(\mathbf{p}, \mathbf{r}), \rho^{i n t}(\mathbf{p}, \mathbf{r})\right] \\
& \mathbf{v} \cdot \partial_{\mathbf{r}} \bar{\rho}^{i n t}(\mathbf{p}, \mathbf{r})=-i\left[-H_{v a c}^{i n t}(p, \mathbf{r})+H_{\nu \nu}^{i n t}(\mathbf{p}, \mathbf{r}), \bar{\rho}^{i n t}(\mathbf{p}, \mathbf{r})\right]
\end{aligned}
$$

\footnotetext{
${ }^{2}$ We assume that the density of $\mu^{ \pm}$and $\tau^{ \pm}$is negligible, and that $\nu_{\mu}$ and $\nu_{\tau}$ feel approximately identical potentials, which have been taken to be zero by convention. An analysis of collective effects including a $\mu-\tau$ potential has recently been carried out [60].
} 
The transformation by $O(\mathbf{r})$ leaves diagonal entries of $\rho(\mathbf{p}, \mathbf{r}), \bar{\rho}(\mathbf{p}, \mathbf{r}), H_{v a c}(p)$ and $H_{\nu \nu}(\mathbf{p}, \mathbf{r})$ unchanged, but the off-diagonal entries become $\mathbf{r}$-dependent. For example, if $V(\mathbf{r})$ varies adiabatically and only in the radial direction, the vacuum Hamiltonian changes according to Eq. (12) as

$$
H_{v a c}^{i n t}(p, r)=H_{v a c}(p)+i r\left[V(r), H_{v a c}(p)\right]+\frac{(i r)^{2}}{2 !}\left[V(r),\left[V(r), H_{v a c}(p)\right]\right]+\ldots .
$$

We know that $V(r)$ is a diagonal matrix, so only the off-diagonal elements of $H_{0}(p)$ are affected by the transformation. The final observables we are going to be interested in, the number fluxes of neutrino flavors, involve only diagonal elements of the density matrix [see Eq. (2)], so the interaction basis is well suited for our purposes.

\section{B. Spherical Symmetry and Single-angle Equations of Motion}

The interaction term $H_{\nu \nu}$ in Eq. (8) depends on $\theta_{\mathbf{p q}}$, i.e. the angle between the momenta of interacting neutrinos. Thus while performing the angular integrals therein, the dependence of the neutrino flux on all angular variables must be taken into account. This makes the problem quite complicated, and an approximate treatment is needed in order to gain useful insights. Two levels of approximation have been considered in literature, viz. multi-angle and single-angle. In the multi-angle approximation, azimuthal symmetry about the axis defined by the source and observer is usually assumed, but not complete spherical symmetry. This essentially captures the effects of correlations between trajectories with different initial launching angles. The effects of such correlations can have interesting consequences which have been explored in detail [29, 41, 42, 4749]. In the single-angle approximation, it is assumed that the flavor evolution does not significantly depend on any of the angular coordinates (i.e. the evolution is spherically symmetric), and thus we can integrate over all angular degrees of freedom trivially. One must then choose a representative value for $\cos \theta_{\mathbf{p}}$, which we take to be $1 / 2$.

We assume half-isotropic emission from a source of radius $r_{0}$, as defined in [47], and write

$$
\begin{aligned}
n_{\nu}(\mathbf{p}, \mathbf{r}) & =n_{\nu}(p, r)=n_{\nu}\left(p, r_{0}\right) r_{0}^{2} / r^{2} \\
\rho(\mathbf{p}, \mathbf{r}) & =\rho(p, r) .
\end{aligned}
$$

In the steady state, the fluxes of neutrinos and antineutrinos can be written as

$$
\begin{aligned}
\Phi_{\nu} & =\int d p 2 \pi p^{2} 4 \pi r_{0}^{2} n_{\nu}\left(p, r_{0}\right), \\
\Phi_{\bar{\nu}} & =\int d p 2 \pi p^{2} 4 \pi r_{0}^{2} \bar{n}_{\nu}\left(p, r_{0}\right),
\end{aligned}
$$


the total flux being $\Phi=\Phi_{\nu}+\Phi_{\bar{\nu}}$.

A further "unification" in the notation for neutrinos and antineutrinos is possible by noting that their equations of motion, i.e. Eqs. (10) and (11), differ only in the sign of $H_{v a c}(p)$. This suggests a change of variables from $p$ to

$$
\omega=\left|\Delta m_{13}^{2}\right| /(2 p)
$$

Using the same convention as [34], we define for neutrinos

$$
n_{\nu}(\omega, r) \equiv n_{\nu}(p(\omega), r), \quad \rho(\omega, r) \equiv \rho(p(\omega), r)
$$

and for antineutrinos

$$
n_{\nu}(-\omega, r) \equiv \bar{n}_{\nu}(p(\omega), r), \quad \rho(-\omega, r) \equiv \bar{\rho}(p(\omega), r)
$$

The negative values of $\omega$ thus correspond to antineutrinos. Then we need to solve only for $\rho(\omega, r)$, albeit at the cost of extending the domain of $\omega$ to both positive and negative values. This simplifies the $H_{\nu \nu}(\mathbf{p}, \mathbf{r})$ term in Eq. (8) to $^{3}$

$$
H_{\nu \nu}(r)=\mu(r) \int_{-\infty}^{\infty} d \omega f(\omega) \rho(\omega, r) \operatorname{sgn}(\omega)
$$

in terms of the distribution function

$$
f(\omega)=\frac{1}{\Phi} \frac{\left|\Delta m_{13}^{2}\right|^{3} \pi^{2} r_{0}^{2}}{\omega^{4}} n_{\nu}\left(\omega, r_{0}\right)
$$

normalized as $\int_{-\infty}^{\infty} d \omega f(\omega)=1$, and the "collective potential"

$$
\mu(r)=\mu_{0} g(r)
$$

Here $\mu_{0}$ is the collective potential at the neutrinosphere:

$$
\mu_{0} \equiv \mu\left(r_{0}\right)=\frac{3 \sqrt{2} G_{F} \Phi}{128 \pi^{4} r_{0}^{2}},
$$

and the "geometric dilution factor" $g(r)$ is given by

$$
\begin{aligned}
g(r) & \left.\equiv \frac{4 r_{0}^{2}}{3 r^{2}} \int_{\sqrt{1-\left(r_{0} / r\right)^{2}}}^{1} d\left(\cos \theta_{\mathbf{q}}\right)\left(1-\cos \theta_{\mathbf{q}} \cos \theta_{\mathbf{p}}\right)\right|_{\cos \theta_{\mathbf{p}}=1 / 2} \\
& =\frac{4 r_{0}^{2}}{3 r^{2}}\left(1-\sqrt{1-\frac{r_{0}^{2}}{r^{2}}}-\frac{r_{0}^{2}}{4 r^{2}}\right)
\end{aligned}
$$

\footnotetext{
3 Note that $H_{\nu \nu}(\mathbf{p}, \mathbf{r})$ depended on $\mathbf{p}$ only through the direction of $\mathbf{p}$. This dependence no longer survives in the single-angle approximation.
} 
The geometric dilution factor equals unity for $r=r_{0}$, whereas at large $r_{0}$, it decreases as $1 / r^{4}$. The decrease of neutrino densities from a finite source accounts for a factor of $1 / r^{2}$, whereas the additional dilution factor of approximately $1 / r^{2}$ arises from the integral in Eq. (28), due to the decreasing angle subtended by the source and reduced collinearity, which are encoded in the limits and the integrand respectively [43]. Note that the exact numerical factors depend on the choice of $\cos \theta_{\mathbf{p}}$

The total flux $\Phi$ remains conserved as long as there is no explicit temporal variation of the overall luminosity. We work in the steady state approximation and assume the luminosity to be constant in time. Slow variations in it may be taken into account by including an additional time dependent factor. Note that $f(\omega)$ is independent of $r$, which embodies the fact that the normalized neutrino spectrum does not change. Using Eq. (2), we can also write the flavor dependent $\omega$-spectra $f_{\nu_{\alpha}}(\omega, r)$ as

$$
f_{\nu_{\alpha}}(\omega, r)=f(\omega) \rho_{\nu_{\alpha} \nu_{\alpha}}(\omega, r)
$$

Note that $f_{\nu_{e}}(\omega, r)$ contains the spectra of both $\nu_{e}$ and $\bar{\nu}_{e}$, and depends on $r$ only through $\rho_{\nu_{e} \nu_{e}}(\omega, r)$. It would be a constant on the trajectory if there were no flavor evolution of $\rho_{\nu_{e} \nu_{e}}(\omega, r)$. For later use, we define the energy integrated neutrino fluxes for each flavor as

$$
\begin{aligned}
\Phi_{\nu_{e}}(r) & =\Phi \int_{0}^{\infty} d \omega f_{\nu_{e}}(\omega, r), \\
\Phi_{\nu} & =\Phi_{\nu_{e}}(r)+\Phi_{\nu_{x}}(r)+\Phi_{\nu_{y}}(r), \\
\Phi_{\bar{\nu}_{e}}(r) & =\Phi \int_{-\infty}^{0} d \omega f_{\nu_{e}}(\omega, r), \\
\Phi_{\bar{\nu}} & =\Phi_{\bar{\nu}_{e}}(r)+\Phi_{\bar{\nu}_{x}}(r)+\Phi_{\bar{\nu}_{y}}(r) .
\end{aligned}
$$

With these approximations, the problem is reduced to an ordinary one dimensional problem along the radial direction. We denote the derivative with respect to $r$ using a "dot", and using Eqs. (10) and (11), arrive at the single-angle equations of motion

$$
v_{r} \dot{\rho}(\omega, r)=-i\left[+H_{v a c}(\omega, h)+V(r)+H_{\nu \nu}(r), \rho(\omega, r)\right],
$$

where $h \equiv \operatorname{sgn}\left(\Delta m_{13}^{2}\right)$ encodes the dependence on mass hierarchy. Here

$$
v_{r}=\sqrt{1-\frac{r_{0}^{2}}{r^{2}} \sin ^{2} \theta_{\mathbf{p}}\left(r_{0}\right)}
$$

is the radial velocity of the neutrino. Note that for $r \gg r_{0}$, we have $v_{r} \approx 1$. Since the flavor conversions due to collective effects start becoming significant only for $r>4 r_{0}$ [47, 48], for our 
analytic approximations we take $v_{r}=1$, making Eq. (34)

$$
\dot{\rho}(\omega, r)=-i\left[+H_{v a c}(\omega, h)+V(r)+H_{\nu \nu}(r), \rho(\omega, r)\right] .
$$

We have thus used the spherical symmetry of the problem, and the simple energy dependence, to rephrase the equations of motion in a somewhat simpler form. This single-angle approximation is probably crude, but it has been shown in numerical simulations (for two flavors) that this approximation seems to work reasonably well [48]. It also seems that the multi-angle effects are suppressed when the neutrino and antineutrino spectra are not identical [47]. We assume the above results to hold true for three flavors as well, and ignore multi-angle effects in this work. Thus, for an analytical understanding of various flavor conversion phenomena associated with this system, we confine our discussion to the steady-state single-angle half-isotropic approximation that we have outlined above.

\section{Bloch Vector Notation}

In the single-angle approximation, it is useful to re-express the density matrices and the Hamiltonian as Bloch vectors. The idea, analogous to the two-flavor case, is to express the matrices in a basis of hermitian matrices, and to study the motion of the vectors constructed out of the expansion coefficients (which are called the Bloch vectors). In our problem, we choose the basis consisting of the $3 \times 3$ identity matrix $I$, and the 8 Gell-Mann matrices $\Lambda_{a}$ given by

$$
\begin{aligned}
\Lambda_{1}= & {\left[\begin{array}{lll}
0 & 1 & 0 \\
1 & 0 & 0 \\
0 & 0 & 0
\end{array}\right], \Lambda_{2}=\left[\begin{array}{ccc}
0 & -i & 0 \\
i & 0 & 0 \\
0 & 0 & 0
\end{array}\right], \Lambda_{3}=\left[\begin{array}{ccc}
1 & 0 & 0 \\
0 & -1 & 0 \\
0 & 0 & 0
\end{array}\right], } \\
\Lambda_{4}= & {\left[\begin{array}{lll}
0 & 0 & 1 \\
0 & 0 & 0 \\
1 & 0 & 0
\end{array}\right], \Lambda_{5}=\left[\begin{array}{ccc}
0 & 0 & -i \\
0 & 0 & 0 \\
i & 0 & 0
\end{array}\right], \Lambda_{6}=\left[\begin{array}{lll}
0 & 0 & 0 \\
0 & 0 & 1 \\
0 & 1 & 0
\end{array}\right], } \\
\Lambda_{7}= & {\left[\begin{array}{lll}
0 & 0 & 0 \\
0 & 0 & -i \\
0 & i & 0
\end{array}\right], \Lambda_{8}=\frac{1}{\sqrt{3}}\left[\begin{array}{ccc}
1 & 0 & 0 \\
0 & 1 & 0 \\
0 & 0 & -2
\end{array}\right], }
\end{aligned}
$$

which satisfy the $S U(3)$ Lie algebra

$$
\left[\Lambda_{a}, \Lambda_{b}\right]=i f_{a b c} \Lambda_{c}
$$


where $a, b, c$ are integers from 1 to 8 . Note that the normalization for the matrices is chosen such that

$$
\operatorname{Tr}\left(\Lambda_{a} \Lambda_{b}\right)=2 \delta_{a b}
$$

The structure constants $f_{a b c}$ are antisymmetric under exchange of any two indices and are specified by

$$
f_{123}=2 ; \quad f_{147}, f_{165}, f_{246}, f_{257}, f_{345}, f_{376}=1 ; \quad f_{678}, f_{458}=\sqrt{3}
$$

Note that basis of traceless matrices $\Lambda_{a}$ can be expressed as a semi-direct sum of

$$
\mathbb{K}=\left\{\Lambda_{1}, \Lambda_{2}, \Lambda_{3}, \Lambda_{8}\right\} \quad \text { and } \quad \mathbb{Q}=\left\{\Lambda_{4}, \Lambda_{5}, \Lambda_{6}, \Lambda_{7}\right\}
$$

i.e. for $K_{a} \in \mathbb{K}$ and $Q_{a} \in \mathbb{Q}$ we have

$$
\left[K_{a}, Q_{b}\right] \in \mathbb{K},\left[Q_{a}, Q_{b}\right] \in \mathbb{K} \quad \text { and } \quad\left[Q_{a}, K_{b}\right] \in \mathbb{Q}
$$

In fact this is not the only choice of $\mathbb{K}$ and $\mathbb{Q}$ that has this property. In addition to the decomposition

$$
\mathbb{K}^{e x}=\left\{\Lambda_{1}, \Lambda_{2}, \Lambda_{3}, \Lambda_{8}\right\} \quad \text { and } \quad \mathbb{Q}^{e x}=\left\{\Lambda_{4}, \Lambda_{5}, \Lambda_{6}, \Lambda_{7}\right\}
$$

as above, we could also choose

$$
\begin{array}{lll}
\mathbb{K}^{e y}=\left\{\Lambda_{3}, \Lambda_{4}, \Lambda_{5}, \Lambda_{8}\right\} & \text { and } \quad \mathbb{Q}^{e y}=\left\{\Lambda_{1}, \Lambda_{2}, \Lambda_{6}, \Lambda_{7}\right\} \quad \text { or } \\
\mathbb{K}^{x y}=\left\{\Lambda_{3}, \Lambda_{6}, \Lambda_{7}, \Lambda_{8}\right\} & \text { and } \quad \mathbb{Q}^{x y}=\left\{\Lambda_{1}, \Lambda_{2}, \Lambda_{4}, \Lambda_{5}\right\} &
\end{array}
$$

which satisfy the conditions in Eq. (42). The meaning of the superscripts $(e x, e y, x y)$ on $\mathbb{K}$ and $\mathbb{Q}$ will become clear later.

Using the basis matrices $I$ and $\Lambda_{a}$, we now express any $3 \times 3$ hermitian matrix $X$ as a vector $\mathbf{X}$ in the $S U(3)$ generator space (with unit vectors $\hat{\mathbf{e}}_{i}$ ) as

$$
X=\frac{1}{3} \mathrm{X}_{0} I+\frac{1}{2} \mathbf{X} \cdot \boldsymbol{\Lambda}
$$

We call $\mathbf{X}$ the Bloch vector corresponding to the matrix $X$. The vector $\mathbf{X}$ must lie completely within an eight-dimensional compact volume, called the Bloch ball, whose various two-dimensional sections are shown in Fig. 1, We say that the vector $\mathbf{X}$ is contained in $\mathbb{K}^{e x}\left(\mathbb{K}^{e y}, \mathbb{K}^{x y}\right)$ if the matrix $X$ can be expressed solely as a linear combination of $\Lambda_{a} \in \mathbb{K}^{e x}\left(\mathbb{K}^{e y}, \mathbb{K}^{x y}\right)$. 


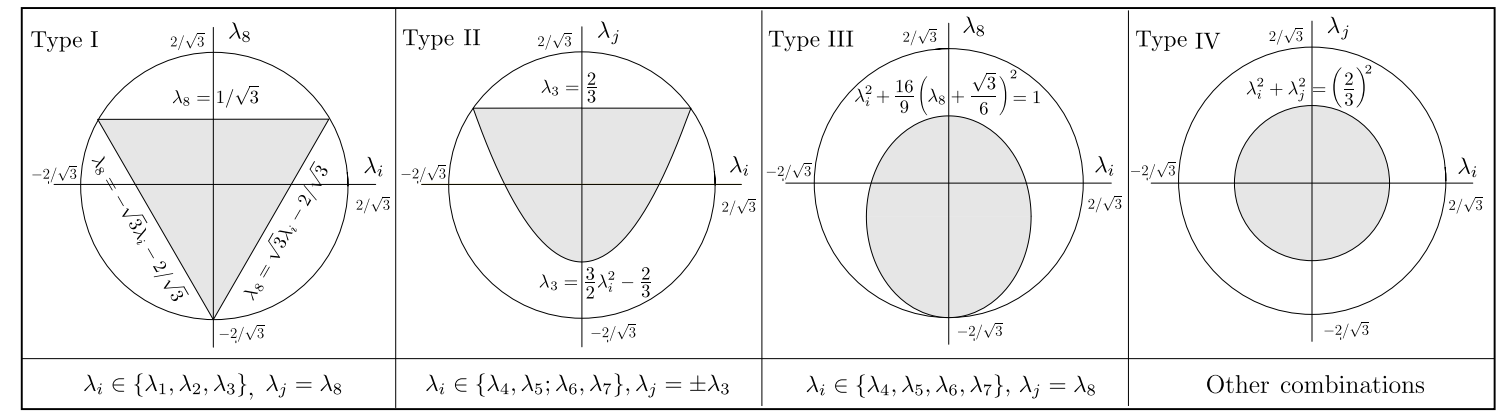

FIG. 1: The shape of the Bloch-ball for a vector $\lambda_{i} \hat{\mathbf{e}}_{i}$ (Figure taken from [62]).

We reparametrize our equations using Eq. (46), and define the Bloch vectors corresponding to the density matrices as

$$
\rho(\omega, r)=\frac{1}{3} \mathrm{P}_{0}(\omega, r) I+\frac{1}{2} \mathbf{P}(\omega, r) \cdot \boldsymbol{\Lambda}
$$

Note that $\boldsymbol{\Lambda}$ is an eight-vector of $3 \times 3$ matrices. The scalar $\mathrm{P}_{0}(\omega, r)$ and the polarization vector $\mathbf{P}(\omega, r)$ encode the flavor content of neutrinos of energy $p=\left|\Delta m_{13}^{2}\right| /(2 \omega)$ at a distance $r$ for $\omega>0$. The negative values of $\omega$ encode the same information for antineutrinos. Since $\rho(\omega, r)$ has been normalised to have unit trace by definition, $\mathrm{P}_{0}(\omega, r)$ is equal to one. We will therefore not worry about the zeroth component of the polarization vector henceforth. For a pure state, $\mathbf{P}(\omega, r)$ lies on the boundary of the shaded region in Fig. 1, and has the magnitude $2 / \sqrt{3}$. For a mixed state, the magnitude of $\mathbf{P}(\omega, r)$ is smaller and the vector lies within the shaded region.

We assume that all neutrinos are produced as flavor eigenstates, i.e. the primary flux consists of $n_{\nu_{\alpha}}\left(p, r_{0}\right)$ and $\bar{n}_{\nu_{\alpha}}\left(p, r_{0}\right)$ with energy $p$. The initial density matrix $\rho\left(p, r_{0}\right)$ is therefore $\operatorname{Diag}\left(n_{\nu_{e}}\left(p, r_{0}\right), n_{\nu_{x}}\left(p, r_{0}\right), n_{\nu_{y}}\left(p, r_{0}\right)\right)$, and similarly for antineutrinos. The initial polarization vector may be written as

$$
\mathbf{P}\left(\omega, r_{0}\right)=\frac{f_{\nu_{e}}\left(\omega, r_{0}\right)-f_{\nu_{x}}\left(\omega, r_{0}\right)}{f(\omega)} \hat{\mathbf{e}}_{3}+\frac{f_{\nu_{e}}(\omega)+f_{\nu_{x}}(\omega)-2 f_{\nu_{y}}\left(\omega, r_{0}\right)}{\sqrt{3} f(\omega)} \hat{\mathbf{e}}_{8} .
$$

The polarization vector $\mathbf{P}(\omega, r)$, when projected onto the $\mathbf{e}_{3}-\mathbf{e}_{8}$ plane, must lie within the triangle in Fig. 2, where we show a representative $\mathbf{P}(\omega, r)$ projected on the $\mathbf{e}_{3}-\mathbf{e}_{8}$ plane. The pure electron flavor is represented by

$$
\mathbf{e}_{e}=\hat{\mathbf{e}}_{3}+\frac{\hat{\mathbf{e}}_{8}}{\sqrt{3}}
$$

The $\nu_{e}$ or $\bar{\nu}_{e}$ content with energy $p$ at position $r$ is given by

$$
\rho_{\nu_{e} \nu_{e}}(p, r)=\frac{n_{\nu_{e}}(p, r)}{n_{\nu}(p)}=\frac{f_{\nu_{e}}(\omega, r)}{f(\omega)}=\frac{1}{3}+\frac{\mathbf{P} \cdot \mathbf{e}_{e}}{2}=\frac{d_{e}}{\sqrt{3}}
$$




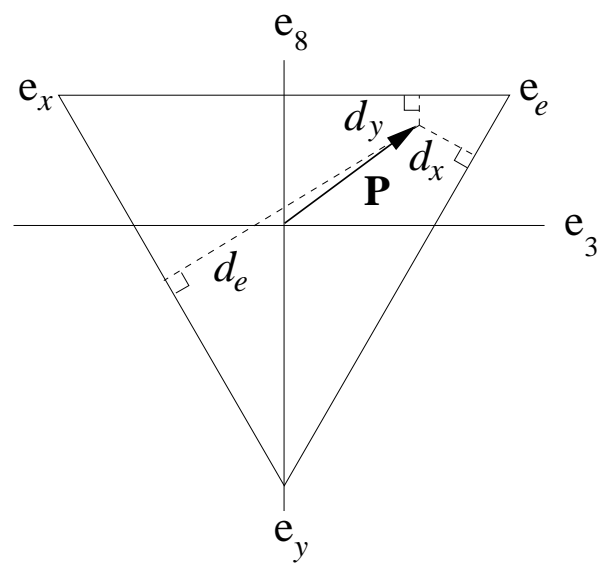

FIG. 2: The projection of a polarization vector $\mathbf{P}$ on the $\mathbf{e}_{3}-\mathbf{e}_{8}$ plane

The projection of $\mathbf{P}$ on $\hat{\mathbf{e}}_{e}$ is thus related to $\rho_{\nu_{e} \nu_{e}}(p, r)=f_{\nu_{e}}(\omega, r) / f(\omega)$ as above. The same quantity can be easily visualized from the figure as $d_{e} / \sqrt{3}$, where $d_{e}$ is the distance of the tip of $\mathbf{P}$ from the side of the triangle that is perpendicular to $\hat{\mathbf{e}}_{e}$ (as shown in the figure). The number of $\nu_{x}$ and $\nu_{y}$ are also similarly calculated. Negative values of $\omega$ encode the same information for the antineutrinos. This gives a simple pictorial way to represent the flavor content of the ensemble by plotting the tip of the projection of $\mathbf{P}(\omega, r)$ on the $\mathbf{e}_{3}-\mathbf{e}_{8}$ plane. ${ }^{4}$

For the mass term in the Hamiltonian, we have

$$
H_{v a c}(\omega, h)=h \omega\left(\frac{1}{3} \mathrm{~B}_{0} I+\frac{1}{2} \mathbf{B} \cdot \boldsymbol{\Lambda}\right)
$$

where

$$
\begin{aligned}
h \mathbf{B}= & \epsilon c_{13} \sin 2 \theta_{12} \hat{\mathbf{e}}_{1}+\left(s_{13}^{2}-\epsilon\left(c_{12}^{2}-c_{13}^{2} s_{12}^{2}\right)\right) \hat{\mathbf{e}}_{3}+\left(1-\epsilon s_{12}^{2}\right) \sin 2 \theta_{13} \hat{\mathbf{e}}_{4} \\
& -\epsilon s_{13} \sin 2 \theta_{12} \hat{\mathbf{e}}_{6}+\left((-2+\epsilon)\left(3 c_{13}^{2}-1\right)+3 \epsilon s_{13}^{2}\left(2 c_{12}^{2}-1\right)\right) /(2 \sqrt{3}) \hat{\mathbf{e}}_{8} .
\end{aligned}
$$

Note that $\omega$ for neutrinos is always positive in this convention, and the negative sign of $\Delta m_{13}^{2}$ for inverted hierarchy is absorbed into $\mathbf{B}$. The terms involving $\epsilon=\Delta m_{12}^{2} / \Delta m_{13}^{2}$ arise from the mixing of the third flavor, and the three-flavor effects enter through them. The sign of $\epsilon$ is positive if the mass hierarchy is normal $\left(\Delta m_{13}^{2}>0\right)$ and negative otherwise. This, along with the overall sign due to $h$, guarantees that the contributions from $\Delta m_{12}^{2}$ always have the same sign. Note that $\mathrm{B}_{2}, \mathrm{~B}_{5}, \mathrm{~B}_{7}$ vanish in the absence of $C P$-violation.

\footnotetext{
${ }^{4}$ Note that probability conservation in this representation corresponds to the theorem that the sum of the lengths of perpendiculars dropped from any point inside an equilateral triangle to the three sides is a constant.
} 
The MSW potential defined in Eq. (7) may be represented as

$$
V(r)=\lambda(r)\left(\frac{1}{3} \mathrm{~L}_{0} I+\frac{1}{2} \mathbf{L} \cdot \boldsymbol{\Lambda}\right),
$$

where $\lambda(r)=\sqrt{2} G_{F} n_{e^{-}}(r)$. The vector $\mathbf{L}$ parameterizes the effect of background electrons, and is given by

$$
\mathbf{L}=\hat{\mathbf{e}}_{3}+\hat{\mathbf{e}}_{8} / \sqrt{3}
$$

The $H_{\nu \nu}(r)$ term defined in Eq. (24) can also be simply written as

$$
H_{\nu \nu}(r)=\mu(r)\left(\frac{1}{3} \mathrm{D}_{0} I+\frac{1}{2} \mathbf{D}(r) \cdot \boldsymbol{\Lambda}\right)
$$

where $\mathbf{D}(r)$ is defined as

$$
\mathbf{D}(r)=\int d \omega f(\omega) \mathbf{P}(\omega, r) \operatorname{sgn}(\omega)
$$

In the next section, we shall express the evolution equation, i.e. Eq. (36) in terms of the Bloch vectors $\mathbf{P}(\omega, r), \mathbf{B}(\omega, h), \mathbf{L}$ and $\mathbf{D}(r)$.

\section{Generalized Gyroscope Equations}

We have expressed our problem in terms of the eight-dimensional Bloch vectors, and now we shall see that the equations of motion formally resemble the equations of a gyroscope. To make this apparent, we define $\times$ as a generalized "cross product" [63] with $f_{a b c}$ as the structure constants, instead of the usual $\epsilon_{a b c}$ that appears in the two-flavor approximation, e.g.

$$
\mathbf{B} \times \mathbf{P} \equiv \sum_{a, b=1}^{8} f_{a b c} \mathrm{~B}_{a} \mathrm{P}_{b} \hat{\mathbf{e}}_{c} .
$$

This makes it possible to write the equations of motion, i.e. Eq. (36), compactly as

$$
\dot{\mathbf{P}}(\omega, r)=(\omega \mathbf{B}+\lambda(r) \mathbf{L}+\mu(r) \mathbf{D}(r)) \times \mathbf{P}(\omega, r) \equiv \mathbf{H}(\omega, r) \times \mathbf{P}(\omega, r),
$$

where $\mathbf{P}(\omega, r), \mathbf{B}, \mathbf{L}, \mathbf{D}(r)$ are defined in Eqs. (47), (52), (54) and (56) respectively. The couplings $\omega, \mu(r)$ and $\lambda(r)$ are defined in Eqs. (21), (28) and (53) respectively. Equation (58) resembles the equation of a spin in an external magnetic field, or equivalently, that of a gyroscope. We must remember that this similarity is purely formal, because unlike in the two-flavor case, we cannot write an arbitrary Bloch vector as a linear combination of two Bloch vectors and their cross product. 
We shall show in Sec. IIE that under certain approximations these generalized gyrosope equations can be given a geometrical interpretation.

The effects of the matter term $\lambda(r) \mathbf{L}$ in Eq. (58) can be rotated away by going to the interaction frame as described in Eq. (12), where a matrix $A$ becomes $A^{i n t}=O A O^{-1}$. In order to determine the Bloch vector corresponding to $A^{\text {int }}$, we equate

$$
\frac{\mathrm{A}_{0}}{3} I+\sum_{a=1}^{8} \frac{\mathrm{A}_{a} \Lambda_{a}}{2}=O A O^{-1} .
$$

Multiplying both sides by $\Lambda_{a}$ and taking trace, we get

$$
\mathrm{A}_{a}^{i n t}=\operatorname{Tr}\left(\Lambda_{a} O A O^{-1}\right)
$$

where we have used $\operatorname{Tr}\left(\Lambda_{a} \Lambda_{b}\right)=2 \delta_{a b}$. In particular, the Bloch vector $\mathbf{B}^{\text {int }}$ may be written using Eqs. (13) and (60) as

$$
\begin{aligned}
\mathbf{B}^{i n t}(r)= & \mathrm{B}_{1} \cos \zeta(r) \hat{\mathbf{e}}_{1}+\mathrm{B}_{1} \sin \zeta(r) \hat{\mathbf{e}}_{2}+\mathrm{B}_{3} \hat{\mathbf{e}}_{3} \\
& +\mathrm{B}_{4} \cos \zeta(r) \hat{\mathbf{e}}_{4}+\mathrm{B}_{4} \sin \zeta(r) \hat{\mathbf{e}}_{5}+\mathrm{B}_{6} \hat{\mathbf{e}}_{6}+\mathrm{B}_{7} \hat{\mathbf{e}}_{7}+\mathrm{B}_{8} \hat{\mathbf{e}}_{8},
\end{aligned}
$$

where $\zeta(r)=\int_{0}^{r} V\left(r^{\prime}\right) d r^{\prime}$. In dense matter, $\mathrm{B}_{a}^{i n t}(r)$ oscillates rapidly with the frequency $\sim V(r)$, mimicking a suppression in the relevant mixing angles as in the two-flavor case [32].

We also define the "signed" and "unsigned" $n^{t h}$ moments (with $n \geq 0$ ) of $\mathbf{P}(\omega, r)$ as

$$
\begin{aligned}
\mathbf{D}^{(n)}(r) & =\int d \omega \omega^{n} f(\omega) \mathbf{P}(\omega, r) \operatorname{sgn}(\omega), \\
\mathbf{S}^{(n)}(r) & =\int d \omega \omega^{n} f(\omega) \mathbf{P}(\omega, r) .
\end{aligned}
$$

Note that $\mathbf{D}^{(0)}$ is same as $\mathbf{D}$, and we will therefore refer to $\mathbf{S}^{(0)}$ as $\mathbf{S}$. The evolution of these moments are governed by

$$
\begin{aligned}
\dot{\mathbf{D}}^{(n)}(r) & =\mathbf{B} \times \mathbf{D}^{(n+1)}(r)+(\lambda(r) \mathbf{L}+\mu(r) \mathbf{D}(r)) \times \mathbf{D}^{(n)}(r), \\
\dot{\mathbf{S}}^{(n)}(r) & =\mathbf{B} \times \mathbf{S}^{(n+1)}(r)+(\lambda(r) \mathbf{L}+\mu(r) \mathbf{D}(r)) \times \mathbf{S}^{(n)}(r) .
\end{aligned}
$$

We see that the higher moments turn up in equations of motion the lower moments. If we take the dot product of Eq. (64) with $\mathbf{D}^{(n)}(r)$, and of Eq. (65) with $\mathbf{S}^{(n)}(r)$, we get

$$
\begin{aligned}
\partial_{r}\left|\mathbf{D}^{(n)}(r)\right|^{2} & =\mathbf{D}^{(n)}(r) \cdot \mathbf{B} \times \mathbf{D}^{(n+1)}(r), \\
\partial_{r}\left|\mathbf{S}^{(n)}(r)\right|^{2} & =\mathbf{S}^{(n)}(r) \cdot \mathbf{B} \times \mathbf{S}^{(n+1)}(r) .
\end{aligned}
$$

The above dependence of the moments on $r$ implies that there is likely to be a redistribution of flavor as a function of $\omega$. It will be interesting to investigate if these moment equations can be used to predict the nature of the redistribution of flavor spectra. 


\section{E. Heavy-Light factorization of dynamics}

The three-flavor dynamics in the traditional matter-driven scenario can be factorized into the so-called "heavy" and "light" MSW resonances that occur at densities corresponding to $\Delta m_{\text {atm }}^{2}$ and $\Delta m_{\odot}^{2}$ respectively. Appropriate combination of the effective two-flavor dynamics in these two sectors approximates the three-flavor result reasonably well. We now proceed to illustrate a similar simplification for collective effects as well Let us first introduce the notion of "heavy" and "light" subspaces of the Bloch-sphere. In the $\mathbb{K}-\mathbb{Q}$ decomposition shown in Eq. (44), the vectors contained in $\mathbb{K}^{e y}$ are termed "heavy" $(H)$ whereas those contained in $\mathbb{Q}^{e y}$ are termed "light" $(L)$. A general vector $\mathbf{X}$ may be decomposed as

$$
\mathbf{X}=\mathbf{X}^{H}+\mathbf{X}^{L}
$$

In particular, $\mathbf{B}$ in Eq. (52) may be expressed as $\mathbf{B}=\mathbf{B}^{H}+\mathbf{B}^{L}$, with

$$
\begin{aligned}
h \mathbf{B}^{H}= & \left(s_{13}^{2}-\epsilon\left(c_{12}^{2}-c_{13}^{2} s_{12}^{2}\right)\right) \hat{\mathbf{e}}_{3}+\left(1-\epsilon s_{12}^{2}\right) \sin 2 \theta_{13} \hat{\mathbf{e}}_{4} \\
& +\left((-2+\epsilon)\left(3 c_{13}^{2}-1\right)+3 \epsilon s_{13}^{2}\left(2 c_{12}^{2}-1\right)\right) /(2 \sqrt{3}) \hat{\mathbf{e}}_{8}, \\
h \mathbf{B}^{L}= & \epsilon c_{13} \sin 2 \theta_{12} \hat{\mathbf{e}}_{1}-\epsilon s_{13} \sin 2 \theta_{12} \hat{\mathbf{e}}_{6} .
\end{aligned}
$$

The component $\mathbf{B}^{H}$ appears primarily due to $\Delta m_{13}^{2}$, and the other component $\mathbf{B}^{L}$ vanishes if $\epsilon=0$. Note that for two-flavors, or equivalently in the $\epsilon=0$ limit, $\mathbf{B}$ is completely contained in $\mathbb{K}^{e y}$. Now, note the following structure in the equations of motion of a polarization vector:

$$
\begin{aligned}
\dot{\mathbf{P}}^{H}(\omega, r) & =\mathbf{H}^{H}(\omega, r) \times \mathbf{P}^{H}(\omega, r)+\mathbf{H}^{L}(\omega, r) \times \mathbf{P}^{L}(\omega, r), \\
\dot{\mathbf{P}}^{L}(\omega, r) & =\mathbf{H}^{L}(\omega, r) \times \mathbf{P}^{H}(\omega, r)+\mathbf{H}^{H}(\omega, r) \times \mathbf{P}^{L}(\omega, r) .
\end{aligned}
$$

It is clear from the above set of equations that if $\epsilon=0$ and one begins with $\mathbf{P}$ contained in $\mathbb{K}^{e y}$, then $\mathbf{P}$ always remains in $\mathbb{K}^{e y}$, i.e. $\mathbf{P}^{L}(\omega, r)=0$ identically. To investigate this case more closely, we write Eq. (69) for each component of $\mathbf{P}^{H}$ as ${ }^{5}$

$$
\begin{aligned}
& \dot{\mathrm{P}}_{3}=\mathrm{H}_{4} \mathrm{P}_{5}-\mathrm{H}_{5} \mathrm{P}_{4}, \\
& \dot{\mathrm{P}}_{4}=\mathrm{H}_{5} \mathrm{P}_{3}-\mathrm{H}_{3} \mathrm{P}_{5}+\sqrt{3}\left(\mathrm{H}_{5} \mathrm{P}_{8}-\mathrm{H}_{8} \mathrm{P}_{5}\right), \\
& \dot{\mathrm{P}}_{5}=\mathrm{H}_{3} \mathrm{P}_{4}-\mathrm{H}_{4} \mathrm{P}_{3}+\sqrt{3}\left(\mathrm{H}_{8} \mathrm{P}_{4}-\mathrm{H}_{4} \mathrm{P}_{8}\right), \\
& \dot{\mathrm{P}}_{8}=\sqrt{3}\left(\mathrm{H}_{4} \mathrm{P}_{5}-\mathrm{H}_{5} \mathrm{P}_{4}\right) .
\end{aligned}
$$

\footnotetext{
${ }^{5}$ In the following sections, the dependence of the Bloch vectors and the parameters on $\omega$ and $r$ is implicit.
} 
Note that $\dot{\mathrm{P}}_{8}=\sqrt{3} \dot{\mathrm{P}}_{3}$. This suggests that we could rotate our coordinates in the $\mathbf{e}_{3}-\mathbf{e}_{8}$ plane by $-2 \pi / 3$, so that $\widetilde{\mathrm{P}}_{8}$ in the rotated frame becomes a constant of motion. While going to the rotated frame, the components $\mathrm{X}_{3}$ and $\mathrm{X}_{8}$ of any Bloch vector $\mathbf{X}$ transform as

$$
\left(\begin{array}{c}
\widetilde{\mathrm{X}}_{3} \\
\widetilde{\mathrm{X}}_{8}
\end{array}\right)=\left(\begin{array}{cc}
-1 / 2 & -\sqrt{3} / 2 \\
\sqrt{3} / 2 & -1 / 2
\end{array}\right)\left(\begin{array}{c}
\mathrm{X}_{3} \\
\mathrm{X}_{8}
\end{array}\right) .
$$

The other components remain unchanged.

This leads to the following simplified equations of motion for the two-flavor case:

$$
\begin{aligned}
& \dot{\widetilde{\mathrm{P}}}_{3}=-2\left(\mathrm{H}_{4} \mathrm{P}_{5}-\mathrm{H}_{5} \mathrm{P}_{4}\right) \\
& \dot{\widetilde{\mathrm{P}}}_{4}=-2\left(\mathrm{H}_{5} \widetilde{\mathrm{P}}_{3}-\widetilde{\mathrm{H}}_{3} \mathrm{P}_{5}\right) \\
& \dot{\widetilde{\mathrm{P}}}_{5}=-2\left(\widetilde{\mathrm{H}}_{3} \mathrm{P}_{4}-\mathrm{H}_{4} \widetilde{\mathrm{P}}_{3}\right) \\
& \dot{\widetilde{\mathrm{P}}}_{8}=0 .
\end{aligned}
$$

This is the two-flavor limit, where the state $\nu_{x}$ does not participate in the evolution. This is a consequence of all the polarization vectors initially being contained in $\mathbb{K}^{e y}$. The rotated "tilde" frame can therefore be called as the " $e-y$ " frame.

The Eqs. (76), (77) and (78) can be simply written as

$$
\dot{\mathbf{P}}^{e y}=\mathbf{H}^{e y} \times \mathbf{P}^{e y},
$$

where the " $\times$ " can now be taken to be the usual cross product in a three-dimensional space spanned by $\left\{\mathbf{e}_{3}^{e y}, \mathbf{e}_{4}, \mathbf{e}_{5}\right\}$. This clearly exhibits the "gyration" of $\mathbf{P}$ about $\mathbf{H}$, while the component of $\mathbf{P}$ along $\mathbf{e}_{8}^{e y}$ remains constant. The projection of $\mathbf{P}$ changes only along $\mathbf{e}_{3}^{e y}$, which corresponds to $\nu_{e} \leftrightarrow \nu_{y}$ flavor conversions. The problem is thus reduced to the two-flavor limit, for which analytical solutions have been discussed in literature [31, 32, 34].

In the two-flavor limit, it is observed that there are three qualitatively different kinds of motion of the polarization vector in the flavor space. The most familiar case is oscillations in vacuum/matter, where the neutrino-antineutrino density is small $(\mu \ll \omega)$ and each $\mathbf{P}(\omega)$ precesses about $\mathbf{B}$ with frequency $\omega$. The other extreme is when the neutrino-antineutrino density is very large $(\mu \gg \omega)$. In such a situation, all $\mathbf{P}(\omega)$ remain tightly bound together and precess with the average $\omega$ of the ensemble, giving rise to synchronized oscillations. The intermediate regime $(\mu \gtrsim \omega)$ is when the $\mathbf{P}(\omega)$ remain bound together to a large extent, but have a tendency to relax to the state that has the lowest energy. The system is analogous to a pendulum/gyroscope that tries to relax to its vertically downward state, whatever state one might start in. This motion is called bipolar oscillation. 
The motion changes qualitatively and quantitatively with the inclusion of the third flavor. There are two kinds of contribution due to the inclusion of the third flavor. First, we have some extra contributions to $\mathbf{B}^{H}$ that depend on $\epsilon$, which changes the effective values of $\omega$ and $\theta_{13}$. These do not change the motion qualitatively. The second type of contribution is more interesting. It is due to the excursions of the polarization vectors into the $\mathbb{Q}$ subspace under the influence of $\mathbf{B}^{L}$. In particular, the length of $\mathbf{P}^{H}$ is not preserved anymore. To see this clearly, we take the dot product of $\mathbf{P}^{H}$ with Eq. (69) and that of $\mathbf{P}^{L}$ with Eq. (70) to get

$$
\left|\dot{\mathbf{P}}^{H}\right|^{2} / 2=-\left|\dot{\mathbf{P}}^{L}\right|^{2} / 2=\mathbf{P}^{H} \cdot \mathbf{H}^{L} \times \mathbf{P}^{L}
$$

We can clearly see that $\left|\mathbf{P}^{H}\right|$, which was a conserved quantity in the two-flavor case, no longer remains so. The non-conservation is proportional to $\left|\mathbf{H}^{L}\right|$ and $\left|\mathbf{P}^{L}\right|$, both of which go to zero in the two-flavor limit. The addition of the third flavor makes the motion of the projection of $\mathbf{P}$ in the $\mathbf{e}_{3}-\mathbf{e}_{8}$ plane fairly complicated in general, and we shall study it in some interesting regimes in Sec. III.

\section{F. The three-flavor solution}

In this section we extend the method presented in the last section to include the leading corrections due to the mixing of the third flavor. Let us illustrate our prescription in the vacuum limit, where the matter effects as well as the collective effects are neglected. The prescription will later be easily generalized to finite matter densities and significant neutrino-neutrino interactions.

From Eq. (52), the Bloch vector $\mathbf{B}$ may be decomposed as

$$
\omega \mathbf{B}=h \omega \mathbf{B}^{(1)}+h \epsilon \omega \mathbf{B}^{(2)}+h \epsilon \omega s_{13} \mathbf{B}^{(3)}
$$

with

$$
\begin{aligned}
& \mathbf{B}^{(1)}=s_{13}^{2} \hat{\mathbf{e}}_{3}-2\left(3 c_{13}^{2}-1\right) /(2 \sqrt{3}) \hat{\mathbf{e}}_{8}+\left(1-\epsilon s_{12}^{2}\right) \sin 2 \theta_{13} \hat{\mathbf{e}}_{4}, \\
& \mathbf{B}^{(2)}=-\left(c_{12}^{2}-c_{13}^{2} s_{12}^{2}\right) \hat{\mathbf{e}}_{3}+\left(3 c_{13}^{2}-1\right) /(2 \sqrt{3}) \hat{\mathbf{e}}_{8}+c_{13} \sin 2 \theta_{12} \hat{\mathbf{e}}_{1}, \\
& \mathbf{B}^{(3)}=3 s_{13}\left(2 c_{12}^{2}-1\right) /(2 \sqrt{3}) \hat{\mathbf{e}}_{8}-\sin 2 \theta_{12} \hat{\mathbf{e}}_{6} .
\end{aligned}
$$

Note that $\mathbf{B}^{(1)}$ lies completely in $\mathbb{K}^{e y}, \mathbf{B}^{(2)}$ in $\mathbb{K}^{e x}$, and $\mathbf{B}^{(3)}$ in $\mathbb{K}^{x y}$.

In Fig. 3, we show three coordinate frames $e-x, e-y$ and $x-y$ in the $\mathbf{e}_{3}-\mathbf{e}_{8}$ plane. These frames are defined such that, if $\mathbf{P}$ is the projection of $\mathbf{P}$ in the $\mathbf{e}_{3}-\mathbf{e}_{8}$ plane, the components $\mathbf{B}^{(1)}, \mathbf{B}^{(2)}, \mathbf{B}^{(3)}$ in Eq. (85) separately cause $\mathbf{P}$ to move along $\mathbf{e}_{3}^{e y}, \mathbf{e}_{3}^{e x}, \mathbf{e}_{3}^{x y}$ respectively. In order 


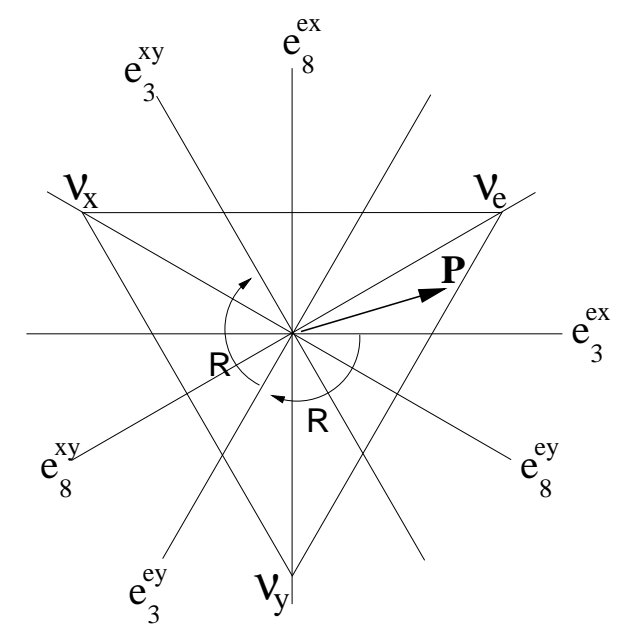

FIG. 3: Useful coordinate choices on $\mathbf{e}_{3}-\mathbf{e}_{8}$ plane

to reduce the motions due to $\mathbf{B}^{(1)}, \mathbf{B}^{(2)}, \mathbf{B}^{(3)}$ separately to two flavor problems as in Sec. IIE, we write

$$
\mathbf{B}^{e y}=\mathrm{R} \mathbf{B}^{(1)}, \quad \mathbf{B}^{e x}=\mathbf{B}^{(2)}, \quad \mathbf{B}^{x y}=\mathrm{R}^{2} \mathbf{B}^{(3)},
$$

where $\mathrm{R}$ is the rotation matrix in Eq. (75) that rotates the $\mathbf{X}_{3}$ and $\mathbf{X}_{8}$ components of a Bloch vector in the $\mathbf{e}_{3}-\mathbf{e}_{8}$ plane by $-2 \pi / 3$. The vectors $\mathbf{B}^{e y}, \mathbf{B}^{e x}, \mathbf{B}^{x y}$ are then simply $\mathbf{B}^{(1)}, \mathbf{B}^{(2)}, \mathbf{B}^{(3)}$ in the frames $e-y, e-x, x-y$ respectively. We can then write Eq. (82) as

$$
\omega \mathbf{B}=\omega^{e y} \mathrm{R}^{-1} \mathbf{B}^{e y}+\omega^{e x} \mathbf{B}^{e x}+\omega^{x y} \mathrm{R}^{-2} \mathbf{B}^{x y}
$$

with the "frequencies" defined as

$$
\omega^{e y}=h \omega \quad \omega^{e x}=h \epsilon \omega \quad \omega^{x y}=h \epsilon \omega s_{13} \sin 2 \theta_{12},
$$

and the "magnetic fields" as

$$
\begin{aligned}
& \mathbf{B}^{e y}=\cos 2 \theta_{13} \hat{\mathbf{e}}_{3}+\left(1-\epsilon s_{12}^{2}\right) \sin 2 \theta_{13} \hat{\mathbf{e}}_{\perp}^{e y}-\left(1-3 s_{13}^{2}\right) /(\sqrt{3}) \hat{\mathbf{e}}_{8} \\
& \mathbf{B}^{e x}=-\left(c_{12}^{2}-c_{13}^{2} s_{12}^{2}\right) \hat{\mathbf{e}}_{3}+c_{13} \sin 2 \theta_{12} \hat{\mathbf{e}}_{\perp}^{e x}+\left(3 c_{13}^{2}-1\right) /(2 \sqrt{3}) \hat{\mathbf{e}}_{8}, \\
& \mathbf{B}^{x y}=-\hat{\mathbf{e}}_{\perp}^{x y}-\sqrt{3} s_{13} \cos 2 \theta_{12} /\left(4 \sin 2 \theta_{12}\right) \hat{\mathbf{e}}_{8} .
\end{aligned}
$$

The vectors $\mathbf{e}_{4}, \mathbf{e}_{1}, \mathbf{e}_{6}$ are the directions transverse to the $\mathbf{e}_{3}-\mathbf{e}_{8}$ plane that are relevant in the three frames, and can be written as $\mathbf{e}_{\perp}^{e y}, \mathbf{e}_{\perp}^{e x}, \mathbf{e}_{\perp}^{x y}$ respectively. The $B^{\alpha \beta}$ are normalized such that $\left|B_{3}^{\alpha \beta}\right|^{2}+\left|B_{\perp}^{\alpha \beta}\right|^{2}=1+\mathcal{O}\left(\epsilon, s_{13}^{2}\right)$. The separate motion due to each $B^{\alpha \beta}$ is then a precession about $B_{3}^{\alpha \beta} \hat{\mathbf{e}}_{3}+B_{\perp}^{\alpha \beta} \hat{\mathbf{e}}_{\perp}$ with a frequency $\omega^{\alpha \beta}$, where the half-angle of the cone is given by $\tan \theta^{\alpha \beta}=$ $\left|B_{\perp}^{\alpha \beta} / B_{3}^{\alpha \beta}\right|$. 
The net motion of the polarization vector may be interpreted as the combination of two-flavor precessions about $\mathbf{e}_{\perp}^{e y}, \mathbf{e}_{\perp}^{e x}$ and $\mathbf{e}_{\perp}^{x y}$ respectively. It can immediately be seen from Eq. (88) that

$$
\left|\omega^{e y}\right| \gg\left|\omega^{e x}\right| \gg\left|\omega^{x y}\right|
$$

i.e. the precession frequencies are hierarchical. Therefore, the motion due to slower frequencies may be neglected over short time scales. More precisely, if we coarse-grain the equation of motion Eq. (58) in $r$ over scales corresponding to $\omega^{e y}$, the effects of $\omega^{e x}$ and $\omega^{x y}$ are negligible. The slowest variation in the solution is due to $\omega^{x y}$, which modulates the faster motion due to $\omega^{e x}$, which in turn modulates the motion at still shorter scales due to $\omega^{e y}$.

Let us denote the evolution of $\mathrm{P}(r)$ under the action of $\mathbf{B}^{e y}, \mathbf{B}^{e x}, \mathbf{B}^{x y}$ by the operators $\mathrm{S}^{e y}(r), \mathrm{S}^{e x}(r), \mathrm{S}^{x y}(r)$ respectively. As long as the condition in Eq. (92) is valid, we can write

$$
\mathrm{P}(r)=\mathrm{S}^{e y}(r) \mathrm{S}^{e x}(r) \mathrm{S}^{x y}(r) \mathrm{P}(0)
$$

where the evolution operators are of the form

$$
\begin{aligned}
& \mathrm{S}^{e y}(r)=\mathrm{R}^{-1}\left(\begin{array}{cc}
\eta\left(\omega^{e y}, \theta^{e y}, \mu, r\right) & 0 \\
0 & 1
\end{array}\right) \mathrm{R}, \\
& \mathrm{S}^{e x}(r)=\left(\begin{array}{cc}
\eta\left(\omega^{e x}, \theta^{e x}, \mu, r\right) & 0 \\
0 & 1
\end{array}\right) \text {, } \\
& \mathrm{S}^{x y}(r)=\mathrm{R}^{-2}\left(\begin{array}{cc}
\eta\left(\omega^{x y}, \theta^{x y}, \mu, r\right) & 0 \\
0 & 1
\end{array}\right) \mathrm{R}^{2} .
\end{aligned}
$$

Here $\eta\left(\omega^{\alpha \beta}, \theta^{\alpha \beta}, \mu, r\right)$ are the evolution functions that can be calculated in a two-flavor approximation using the results in previous literature. In general, the frequencies of these evolution functions are determined by $\omega^{\alpha \beta} \mathrm{s}$ and the amplitudes are determined by the effective mixing angle $\theta^{\alpha \beta} \mathrm{s}$. Each evolution operator $\mathrm{S}^{\alpha \beta}$ takes the state $\mathrm{P}$ to the respective $\alpha-\beta$ frame in which $\mathrm{P}_{8}^{\alpha \beta}$ stays constant and $\mathrm{P}_{3}^{\alpha \beta}$ undergoes precession, and brings $\mathrm{P}$ back to the $\mathbf{e}_{3}-\mathbf{e}_{8}$ frame after precession. Note the matrices $\mathrm{S}^{\alpha \beta}$ are not unitary. The order in which they are operated should be such that the slower oscillations effectively act like an amplitude modulation for the faster oscillations.

It is easy to calculate $\rho_{\nu_{e} \nu_{e}}$ using Eq. (50) as

$$
\rho_{\nu_{e} \nu_{e}}(r)=\frac{1}{3}+\frac{\mathrm{P}(r) \cdot \mathbf{e}_{e}}{2}=\frac{1}{3}+\frac{1}{\sqrt{3}}\left(-\frac{\sqrt{3}}{2} \mathrm{P}_{3}^{e y}(r)+\frac{1}{2} \mathrm{P}_{8}^{e y}(r)\right),
$$

where $\mathrm{P}(r)$ is given by Eq. (93), and $\mathrm{P}_{3}^{e y}, \mathrm{P}_{8}^{e y}$ are components along $\mathbf{e}_{3}^{e y}$ and $\mathbf{e}_{8}^{e y}$ respectively. If neglect effects of the slowest frequency $\omega^{x y}$, the expressions for $\mathrm{P}_{3}^{e y}(r)$ and $\mathrm{P}_{8}^{e y}(r)$ may be written 
as

$$
\begin{aligned}
& \mathrm{P}_{3}^{e y}(r)=\eta\left(\omega^{e y}, \theta^{e y}, \mu, r\right)\left(-\frac{1}{2} \eta\left(\omega^{e x}, \theta^{e x}, \mu, r\right) \mathrm{P}_{3}(0)-\frac{\sqrt{3}}{2} \mathrm{P}_{8}(0)\right), \\
& \mathrm{P}_{8}^{e y}(r)=\left(+\frac{\sqrt{3}}{2} \eta\left(\omega^{e x}, \theta^{e x}, \mu, r\right) \mathrm{P}_{3}(0)-\frac{1}{2} \mathrm{P}_{8}(0)\right) .
\end{aligned}
$$

In the presence of ordinary matter and when the collective effects may be neglected, the same prescription stays valid, simply with the replacements

$$
\hat{\mathbf{e}}_{1} \rightarrow \cos \zeta(r) \hat{\mathbf{e}}_{1}+\sin \zeta(r) \hat{\mathbf{e}}_{2}, \quad \hat{\mathbf{e}}_{4} \rightarrow \cos \zeta(r) \hat{\mathbf{e}}_{4}+\sin \zeta(r) \hat{\mathbf{e}}_{5}
$$

with $\zeta(r)=\int_{0}^{r} V\left(r^{\prime}\right) d r^{\prime}$. It may be seen from Eq. (61) that these replacements take $\mathbf{B}$ to $\mathbf{B}^{\text {int }}$, so that the effect of MSW is taken into account by going to the interaction frame. As observed in Sec. IID, fast oscillations with a frequency $\sim V(r)$ will average out the sinusoidal terms, thus decreasing the contribution from the transverse components of $\mathbf{B}^{(i n t) \alpha \beta}$.

When the collective effects dominate, since the collective potential $H_{\nu \nu}(r)$ in Eq. (36) is independent of energy, neutrinos of all energies precess with a common frequency in all the two-flavor subspaces. The motion is therefore similar to the vacuum case discussed above, with the replacement $\omega \rightarrow\langle\omega\rangle$ as given in Sec. IIIB.

We have thus completed our program of expressing three-flavor effects purely in terms of twoflavor effects. The $r$-dependent functions $\eta(r)$ are known analytically for oscillations in vacuum and for synchronized oscillations, where we can explicitly check our ansatz.

In the case of bipolar oscillations, the situation is more complicated since these are not sinusoidal oscillations, rather $\mathbf{P}$ remains almost static for a period of time and swings through the lowest energy state in a rapid burst. As a result, the fast- or slow-ness of bipolar oscillations as compared to the other precessions is time dependent. We therefore can obtain a qualitative understanding of bipolar oscillations in the three neutrino framework, but only a heuristic form of the analytic solution.

\section{FLAVOR CONVERSION MECHANISMS IN THREE-FLAVOR FORMALISM}

In this section we illustrate the three-flavor effects in some simple examples, where we take constant matter density and box-spectra for neutrinos and antineutrinos. We explain the three-flavor features therein analytically using the " $\mathbf{e}_{3}-\mathbf{e}_{8}$ " triangle diagrams. The insights gained thereby will allow us to understand the more complicated flavor conversions in realistic supernova simulations 
in Sec. IV] For our numerical evaluations in this section, we fix $\left|\Delta m_{\mathrm{atm}}^{2}\right|=2.5 \times 10^{-3} \mathrm{eV}^{2}$ and $\theta_{12}=0.6$. We also choose a box-spectrum for the the neutrino flux i.e. constant over the energy range $E=(1-51) \mathrm{MeV}$, and zero elsewhere.

\section{A. Vacuum and MSW oscillations}

We start with looking at neutrino oscillations in vacuum/matter, with no collective effects. Although this situation has been analyzed in literature in great detail, we illustrate it here in order to familiarize the reader with the analysis in terms of $\mathrm{P}_{3}^{e y}, \mathrm{P}_{8}^{e y}$ and the " $\mathbf{e}_{3}-\mathbf{e}_{8}$ " triangle. This triangle, shown in Fig. 4, helps in understanding the three-neutrino features of flavor conversions. The projection of $\mathbf{P}$ on the $\mathbf{e}_{3}-\mathbf{e}_{8}$ plane represents the flavor content, the allowed region being an equilateral triangle. The three vertices of the triangle represent the three states $\nu_{e}, \nu_{x}$ and $\nu_{y}$ (anticlockwise, from top right). States that lie on the edges connecting them are admixtures of only those two flavors. The interior of the triangle represents states that are admixtures of all three flavors. Quantitatively, for any point on the triangle, the fraction of the neutrinos in flavor $\alpha$ is proportional to its distance from the edge opposite to the $\nu_{\alpha}$ vertex, as shown in Eq. (50).

In Fig. 4, we show the quantities $\mathrm{P}_{3}^{e y}, \mathrm{P}_{8}^{e y}$ and $\rho_{\nu_{e} \nu_{e}}$ as functions of the radial coordinate $r$. For illustration, we start with a pure $\nu_{e}$ flavor, which corresponds to $\left(\mathrm{P}_{3}^{e y}, \mathrm{P}_{8}^{e y}\right)=(-1,1 / \sqrt{3})$. The following observations may be made from the figure.

- The oscillation frequencies depend on the neutrino energy. However in the triangle diagram, the locus of $\mathrm{P}$ for all energies is identical for oscillations in vacuum (therefore, the thin and thick lines overlap). Different energies travel along this orbit at different, but constant speeds proportional to $1 / E$. In matter, the mixing angle begins to depend on the energy and thus the orbits are different for different energies.

- The flavour evolution has two main frequency components, The fast oscillations with frequency $\omega=\Delta m_{\text {atm }}^{2} /(2 p)$ and the slower ones with frequency $\epsilon \omega=\Delta m_{\odot}^{2} /(2 p)$.

- If $\omega$ and $\epsilon \omega$ were commensurate, the orbits in the triangle would be closed curves. However, that is a fine-tuned situation. In general, if $\epsilon$ is not rational, the orbits do not close, but drift parallel to themselves periodically. Indeed, the orbits are analogous to the well-known Lissajous figures.

- $\mathrm{P}_{8}^{e y}$ only has slow modes corresponding to the frequency $\epsilon \omega$. These slow oscillations modulate the amplitude of the upper envelope of $\left|\mathrm{P}_{3}^{e y}\right|$ because the maximum value that $\left|\mathrm{P}_{3}^{e y}\right|$ can take 


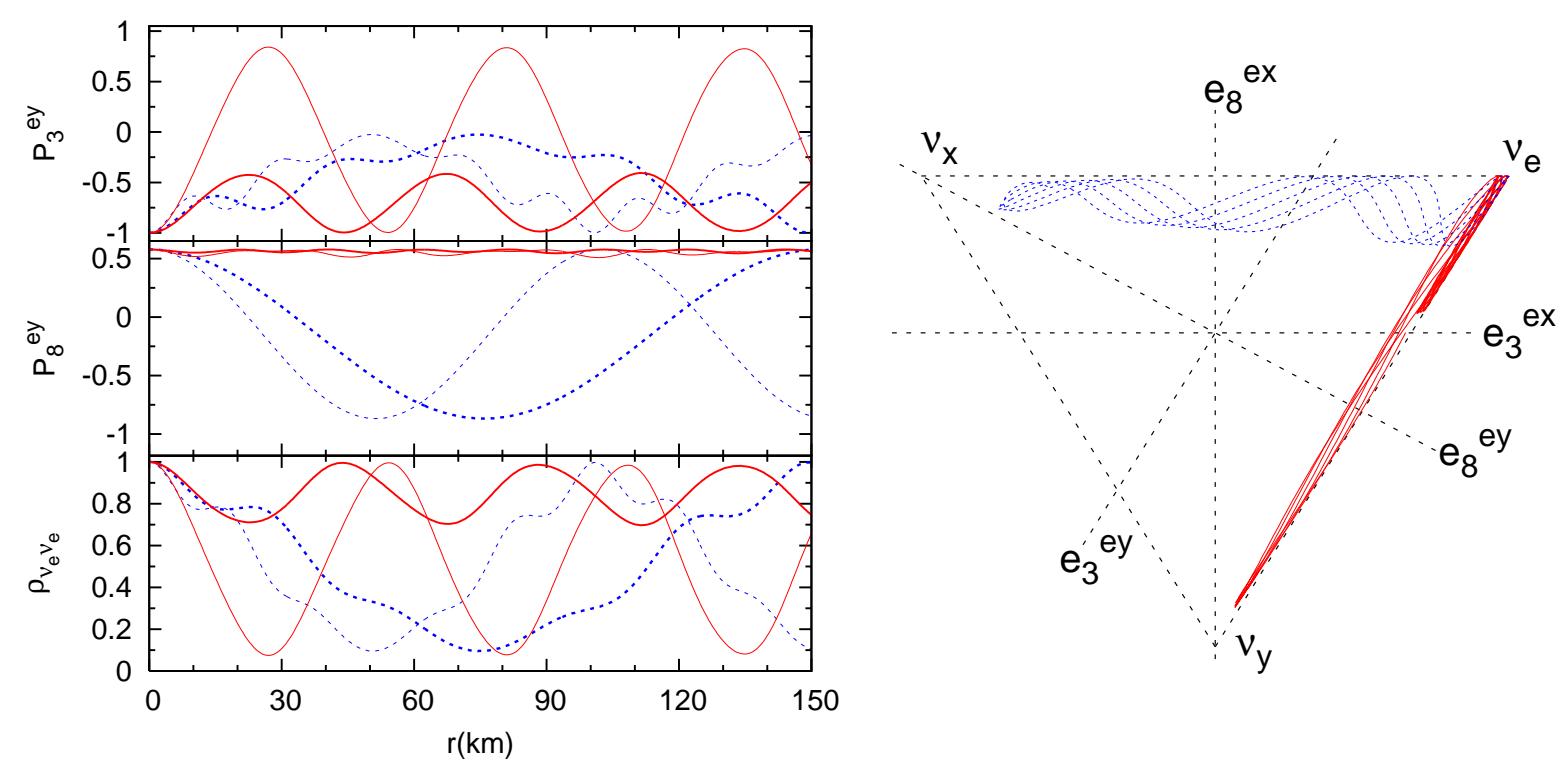

FIG. 4: Neutrino oscillations for $E=20.0 \mathrm{MeV}$ and 29.6 MeV (thin and thick lines respectively). To emphasize the nature the oscillations, we choose $\epsilon=1 / 5.1$ and $\theta_{13}=0.2$. Oscillations in vacuum and matter are shown by dotted (blue) and undotted (red) lines respectively. For matter, we choose normal hierarchy and $\lambda=0.3 \mathrm{~km}^{-1}$.

is reduced when $\mathrm{P}_{8}^{e y}$ deviates from its maximum value of $1 / \sqrt{3}$. The above can be clearly seen from the triangle diagram.

- $\mathrm{P}_{3}^{e y}$ oscillations involve both frequencies, $\omega$ and $\epsilon \omega$. The maximum deviation of $\mathrm{P}_{3}^{e y}$ from unity is governed by the amplitude of modulation of its upper envelop (which depends on $\sin ^{2} 2 \theta_{13}$ ) and the amplitude of faster oscillations superimposed on it (which depends on $\left.\sin ^{2} 2 \theta_{12}\right)$.

- In the two-flavor limit we ignore the mixing with $\nu_{x}$, and as a result $\mathrm{P}_{8}^{e y}$ remains constant. In the triangle, this corresponds to the motion being confined to a line parallel to the $\mathbf{e}_{3}^{e y}$ axis. Indeed, the effect of the third flavor is to extend the motion of $\mathrm{P}$ to the entire triangle, as opposed to only along a line. The deviation of $\mathrm{P}$ from this line quantifies the extent of three-flavor effects.

- The amplitude of oscillations can be read off from the triangle as the extent of the orbit along the $\nu_{e}-\nu_{y}$ edge $\left(2 \sin ^{2} 2 \theta_{13}\right)$ and along the $\nu_{e}-\nu_{x}$ edge $\left(2 \sin ^{2} 2 \theta_{12}\right)$.

- In the presence of matter, mixing angles are suppressed or enhanced depending on the energy and matter density. For $\lambda \sim \epsilon \omega$, the MSW resonance occurs, and the effective mixing angle 
becomes almost maximal, as it happens for the low energy mode shown in the figure. At $\lambda \gg \epsilon \omega$, the state $\nu_{x}$ decouples because of the suppression of the mixing angle in matter, making this an effectively two-flavor $\nu_{e} \leftrightarrow \nu_{y}$ problem. The oscillations in $\mathrm{P}_{8}^{e y}$ have vanishing amplitude and the motion in the triangle is restricted to the $\nu_{e}-\nu_{y}$ edge.

- At even larger matter densities, $\lambda \gg \omega$, the amplitude of $\nu_{e} \leftrightarrow \nu_{y}$ oscillations, which is the amplitude of $\mathrm{P}_{3}^{e y}$ oscillations, starts decreasing and the motion in the triangle becomes more and more confined to be near the $\nu_{e}$ vertex as in the case of the high energy mode shown in the figure.

All the above features may be understood analytically through Eqs. (97)-(99) and the two-flavor evolution functions

$$
\begin{aligned}
& \eta\left(\omega^{e y}, \theta^{e y}, 0, r\right)=1-2 \sin ^{2} 2 \theta_{13} \sin ^{2}\left(\frac{h \omega r}{2}\right), \\
& \eta\left(\omega^{e x}, \theta^{e x}, 0, r\right)=1-2 \sin ^{2} 2 \theta_{12} \sin ^{2}\left(\frac{h \epsilon \omega r}{2}\right) .
\end{aligned}
$$

The above expressions are approximate, since we ignore the slowest frequency modes (depending on $\omega^{x y}$ ) and assume complete factorization. We find however, that these expressions agree with the numerical solution reasonably well .

In the case of finite but constant matter density, we use the angles $\theta^{\alpha \beta}$ and frequencies $\omega^{\alpha \beta}$ in matter, both of which are energy dependent. Note that the amplitudes in this case are proportional to $2 \sin ^{2} 2 \theta^{\alpha \beta}$ in matter and can be maximal (spanning a full edge of the triangle) when there is an MSW resonance.

When the matter density encountered by the neutrino varies such that neutrinos pass through an MSW resonance, they undergo flavor transitions with adiabaticities depending on their energy, the relevant mixing angle and the matter profile. In the limit of a small mixing angle, a completely adiabatic $H$ resonance is represented by a reflection of the neutrino state about $\mathbf{e}_{8}^{e y}$ in the $\mathbf{e}_{3}{ }^{-}$ $\mathbf{e}_{8}$ triangle. A non-adiabatic $H$ resonance corresponds to a state that tries to move towards this reflected point, but does not completely succeed. Passage through the $L$ resonance similarly corresponds to a reflection about $\mathbf{e}_{8}^{e x}$. 


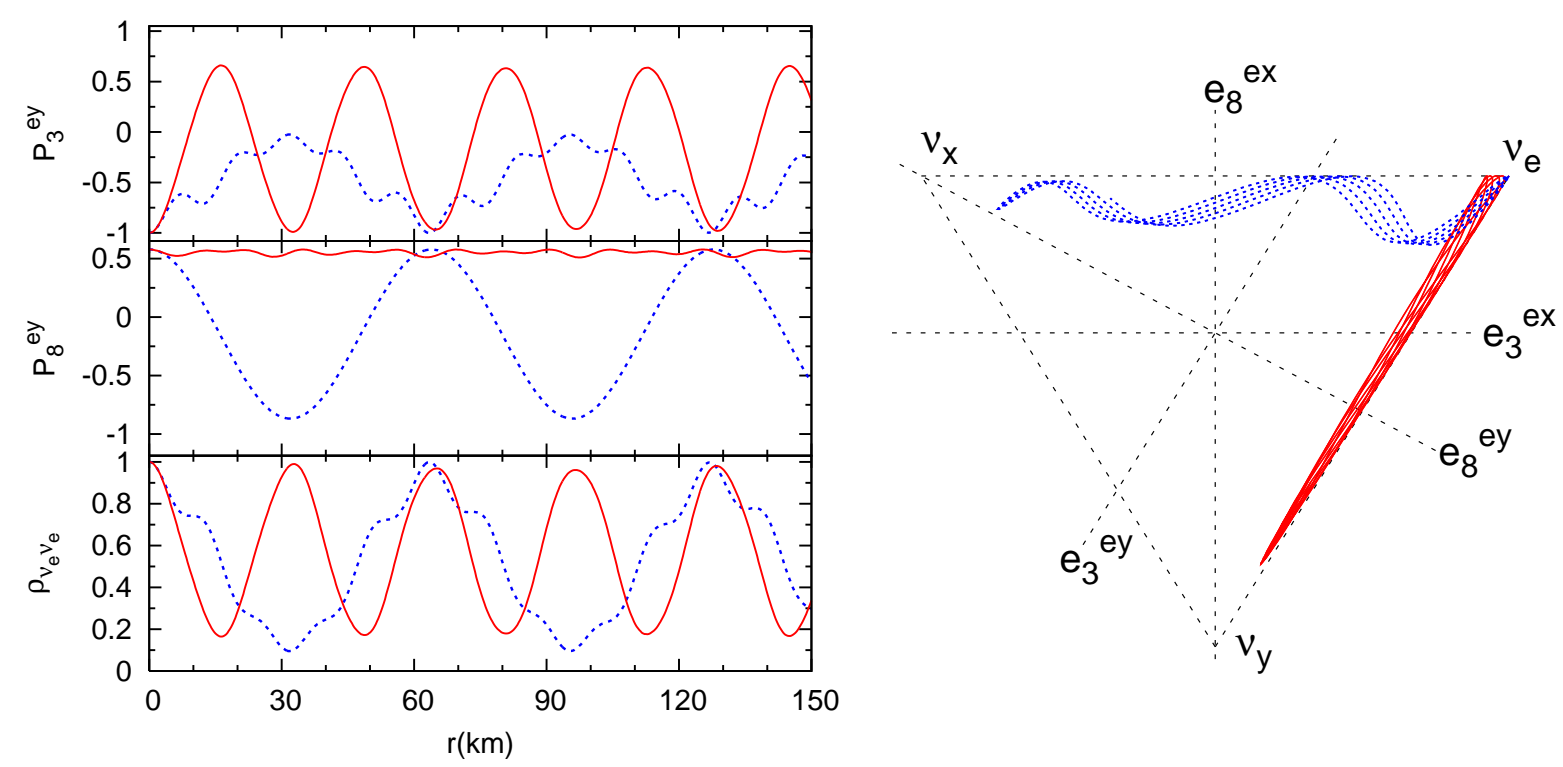

FIG. 5: Synchronized oscillations for neutrinos of $E=20.0$ and $\mathrm{MeV} 29.6 \mathrm{MeV}$, which overlap completely. We choose $\epsilon=1 / 5$ to emphasize the nature of oscillations, $\theta_{13}=0.2$ and $\mu=100 \mathrm{~km}^{-1}$. Oscillations in vacuum and matter are shown by dotted (blue) and undotted (red) lines respectively. For matter, we choose normal hierarchy and $\lambda=0.5 \mathrm{~km}^{-1}$. Note that the orbits on the triangle are the same for different energies.

\section{B. Synchronized oscillations}

At extremely large neutrino densities, it is expected that neutrinos of all energies oscillate synchronously with a common frequency $\left\langle\omega^{\alpha \beta}\right\rangle$ about $\mathbf{B}^{\alpha \beta}$, given in the two-flavor case by [58]

$$
\left\langle\omega^{\alpha \beta}\right\rangle=\frac{\omega^{\alpha \beta}}{\omega} \frac{\mathbf{D} \cdot \mathbf{D}^{(1)}}{|\mathbf{D}|^{2}},
$$

where D's are the moments defined in Eq. (63). The frequency $\left\langle\omega^{\alpha \beta}\right\rangle$ crucially depends on the neutrino energy spectrum. The box-spectrum that we have chosen corresponds to $\left\langle\omega^{e y}\right\rangle \approx 0.49$ $\mathrm{km}^{-1}$. In Fig. 5 we show $\mathrm{P}_{3}^{e y}, \mathrm{P}_{8}^{e y}$ and $\rho_{\nu_{e} \nu_{e}}$ as functions of the radial coordinate $r$ for synchronized neutrino oscillations.

The following observations may be made from the figure:

- The observations in Sec. IIIA remain true, except that neutrinos of all energies oscillate with a common frequency in vacuum in the two flavor limits of each of the $\alpha-\beta$ subspaces. The response of all neutrinos to the neutrino-neutrino potential is thus identical.

- Even in the presence of matter, the synchronized oscillation amplitude is independent of energy, unlike what happens for non-collective oscillations. 
- The amplitude of the slower oscillations is almost maximal because, in the chosen example, $\lambda \sim \epsilon\langle\omega\rangle$.

- The orbits drift periodically, even if $\omega$ and $\epsilon \omega$ are commensurate, because $\langle\omega\rangle$ and $\langle\epsilon \omega\rangle$ are not commensurate in general. This is due to corrections to Eq. (103) arising out of incomplete synchronization.

The above observations are explained analytically along the same lines as the vacuum / MSW case. The two-flavor evolution functions are given by

$$
\begin{aligned}
& \eta\left(\omega^{e y}, \theta^{e y}, 0, r\right)=1-2 \sin ^{2}\left\langle 2 \theta_{13}\right\rangle \sin ^{2}\left(\frac{h\langle\omega\rangle r}{2}\right), \\
& \eta\left(\omega^{e x}, \theta^{e x}, 0, r\right)=1-2 \sin ^{2}\left\langle 2 \theta_{12}\right\rangle \sin ^{2}\left(\frac{h \epsilon\langle\omega\rangle r}{2}\right) .
\end{aligned}
$$

In the plots we see that fast oscillations have wavelength $2 \pi / \omega \approx 12 \mathrm{~km}$. This matches the value of $\left\langle\omega^{e y}\right\rangle$ calculated from Eq. (103).

In the presence of a finite matter density, the MSW potential $\lambda$ also takes an effective average value given by [58]

$$
\langle\lambda\rangle=\lambda \frac{\mathbf{D} \cdot \mathbf{S}}{|\mathbf{D}|^{2}} .
$$

Naturally, the mixing angle is also the same for all energies, since

$$
\sin ^{2}\left\langle 2 \theta^{\alpha \beta}\right\rangle=\frac{\sin ^{2} 2 \theta^{\alpha \beta}}{\left(\langle\lambda\rangle /\left\langle\omega^{\alpha \beta}\right\rangle-\cos 2 \theta^{\alpha \beta}\right)^{2}+\sin ^{2} 2 \theta^{\alpha \beta}} .
$$

Thus not only the frequency, but also the amplitude of oscillations is universal in the synchronized limit. The MSW resonance is collective, occuring with the same adiabaticity for all neutrinos/antineutrinos at the same $\lambda$ when the relevant condition is met, as was shown in the two-flavor case [57-59]. The factorization shown in Sec. IIE allows the result to be extended to the three-flavor situation.

\section{Bipolar Oscillations}

When the hierarchy is inverted and there are comparable numbers of neutrinos and antineutrinos in the system, i.e. $\mu|\mathbf{D}| \sim \omega|\mathbf{B}|$, the influence of the $\omega$ and $\mu$ terms in the equations of motion depends crucially on the relative orientation of $\mathbf{D}, \mathbf{B}$ and the magnitude of $\mathbf{D}$ itself. This subtle interplay gives rise to bipolar oscillations. 
Many of the notions about bipolar oscillations in the two-flavor formalism [32, 33] remain valid with three flavors, since they do not depend on the number of flavors, or equivalently, on the dimensionality of the Bloch vectors. The system is best understood in terms of the "pendulum vector" $\mathbf{Q}$ defined in the interaction frame as 32

$$
\mathbf{Q} \equiv \mathbf{S}-\frac{\omega}{\mu} \mathbf{B}
$$

in terms of which the equations of motion are

$$
\begin{aligned}
& \dot{\mathbf{Q}}=\mu \mathbf{D} \times \mathbf{Q}-\frac{\omega}{\mu} \dot{\mathbf{B}}, \\
& \dot{\mathbf{D}}=\omega \mathbf{B} \times \mathbf{Q} .
\end{aligned}
$$

The antisymmetry of the generalized cross product in Eq. (57) implies that even in the case of three flavors, $|\mathbf{Q}|^{2}$ and $\mathbf{D} \cdot \mathbf{B}$ are conserved for large $\mu$.

In the two-flavor case, the motion can be understood in terms of a spherical pendulum [32], with the total energy given by $\omega \mathbf{B} \cdot \mathbf{Q}+\mu|\mathbf{D}|^{2} / 2$. For normal hierarchy, the pendulum is stable and executes only small oscillations. For inverted hierarchy, however, the system behaves like an inverted pendulum, which tries to relax to its stable position. The polarization vectors then remain almost static, but periodically dip to the configuration with the lowest potential energy B · Q. Thus for inverted hierarchy, one can have a large flavor swap during the dip. The duration between successive dips is given by $\tau^{b i p} \approx \sqrt{\omega \mu|\mathbf{Q}|}$ with logarithmic corrections depending on $\theta$ and $\lambda$. Since $\mu>\omega$, individual $\mathbf{P}$ remain bound to each other, and therefore behave identically to Q.

Addition of a third flavor may change the behaviour significantly, as we show in Figs. 6] and 7 for two extreme values of $\lambda$. We consider the case of inverted hierarchy, and a box-spectrum of energies $E=(1-51) \mathrm{MeV}$ with the number of antineutrinos as $(1-\alpha)$ times the number of neutrinos, with $\alpha=0.2$. Given that the hierarchy in the solar sector is normal, we expect bipolar effect only in the $e-y$ subspace, combined with usual neutrino oscillations in the $e-x$ subspace. The following observations may be made from the figures:

- The evolution of both $\mathrm{P}_{3}^{e y}$ and $\mathrm{P}_{8}^{e y}$ consists of a series of bipolar "kinks" as in the two-flavor case [32], modulated by an envelope with the frequency $\left\langle\omega^{e x}\right\rangle$. The evolutions for neutrinos and antineutrinos closely follow one another, which is expected from the conservation of B $\cdot$ D.

- Significant three-flavor effects are present for small $\lambda$, since the whole triangle is seen to be filled with oscillations, forming a "petal structure" (Fig. 6). It may be interpreted as a 

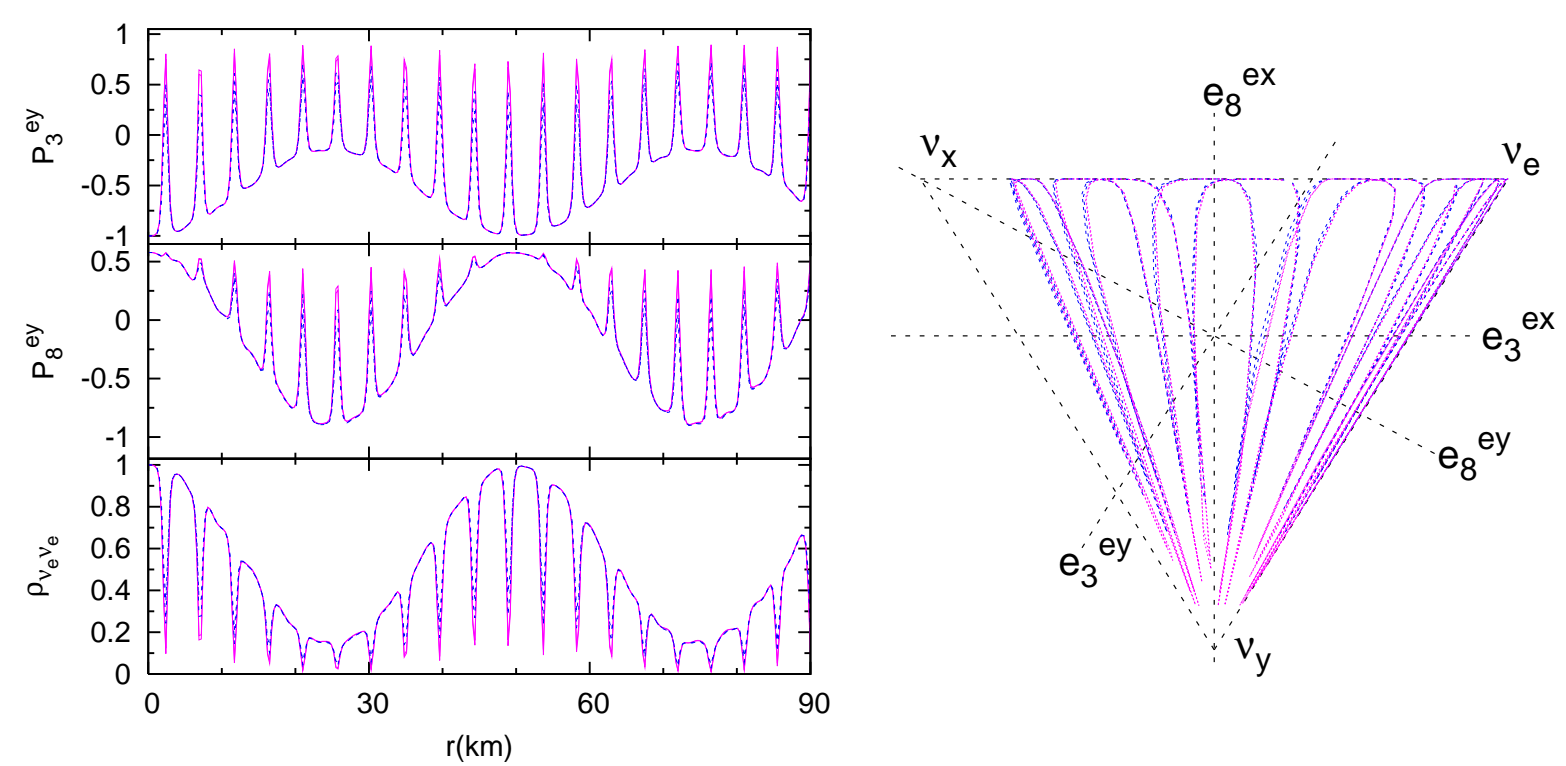

FIG. 6: Bipolar oscillations at small $\lambda$ for neutrinos (dotted, blue) and $20 \%$ fewer antineutrinos (undotted, pink) of different energies, which almost overlap. We choose inverted hierarchy, $|\epsilon|=1 / 30, \theta_{13}=0.01, \mu=$ $10 \mathrm{~km}^{-1}$ and $\lambda=0.001 \mathrm{~km}^{-1}$. Note that the plots are the same for different energies, because of strong collective behaviour.

combination of slow $\nu_{e} \leftrightarrow \nu_{x}$ oscillations and bipolar oscillations that tend to take the state towards $\nu_{y}$ in periodic bursts.

- The extent of motion towards $\nu_{y}$ depends mainly on the asymmetry $\alpha$, whereas that towards $\nu_{x}$ depends on $\sin ^{2} 2 \theta_{12}$.

- For large $\lambda$ (Fig. 7), the oscillations in the $e-x$ sector are suppressed since the effective mixing angle $\theta_{12}$ in matter becomes small. The amplitude of the bipolar motion is however not affected substantially.

Bipolar oscillations (even in the two-flavor limit) do not have a sinusoidal form, hence they are not associated with a fixed frequency. They may be looked upon as a combination of a low frequency (during the time that the $\nu_{y}$ component is stationary, which we shall call the A phase) and a high frequency (the sudden dip towards $\nu_{y}$, which we shall call the B phase). Therefore, our prescription in Sec. IIF has to be applied with care. Note that the order of evolution matrices in Eq. (96) is supposed to be in the decreasing order of frequencies. Even if we neglect the slow evolution due to $\mathbf{B}^{x y}$, strictly speaking during the A phase, one should use the order $\mathbf{S}^{e x} \mathbf{S}^{e y}$ and during the B phase, the order should be $S^{e y} S^{e x}$. However, we find numerically that the evolution $\mathrm{S}^{e x} \mathrm{~S}^{e y}$ closely matches the three-flavor solution over the complete evolution. This therefore may 

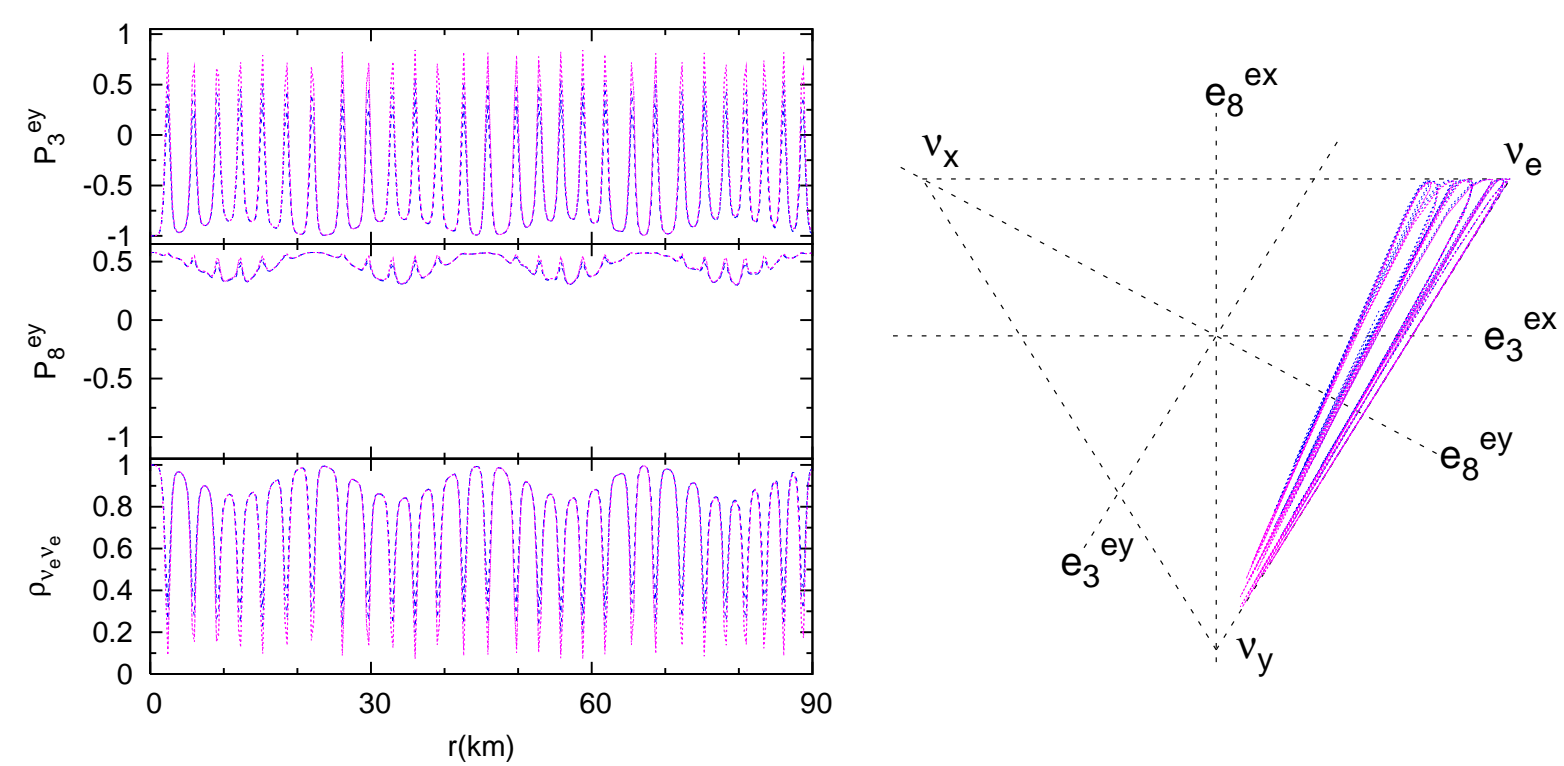

FIG. 7: Bipolar oscillations at large $\lambda$ for neutrinos (dotted, blue) and antineutrinos (undotted, pink) of different energies, which almost overlap. We choose inverted hierarchy, $|\epsilon|=1 / 30, \theta_{13}=0.01, \mu=10 \mathrm{~km}^{-1}$ and $\lambda=0.3 \mathrm{~km}^{-1}$. Note that the plots are the same for different energies, because of strong collective behaviour.

be taken to be the heuristic solution for the bipolar oscillations in the three-flavor case.

We have not considered normal hierarchy, in which we expect that starting with $\nu_{e}$ we'll have a stable system that will not undergo bipolar oscillations, whereas starting with $\nu_{x}$ or $\nu_{y}$, we'll have independent bipolar oscillations towards $\nu_{e}$. It will be interesting to analyze the details of such a scenario, however it is beyond the scope of this paper.

\section{Spectral splitting}

As a system of neutrinos and antineutrinos transits from the collective regime $(\mu \gg \omega)$ to vacuum $(\mu \sim 0)$, the polarization vectors $\mathbf{P}$ keep trying to align with $\mathbf{H}$ in the adiabatic approximation. Due to the conservation of $\mathbf{B} \cdot \mathbf{D}$, as shown in Sec. IIIC, this alignment is not possible for all $\mathbf{P}$. Indeed, neutrinos with high energy need to flip over and anti-align with $\mathbf{B}$ (which equals $\mathbf{H}$ in vacuum) [34, 35]. This leads to a sharp split in the energy spectrum, with the high energy $\nu_{e}$ getting completely converted to the non-electron flavor and vice versa.

A crucial requirement for the splits to develop is the preparation of the sytem for the split by the generation of components of $\mathbf{P}$ that are transverse to $\mathbf{B}$. Bipolar oscillations do this easily for inverted hierarchy, independent of matter effects. For normal hierarchy, in the presence of large matter effects the oscillations are suppressed, but an MSW resonance can prepare the transverse 

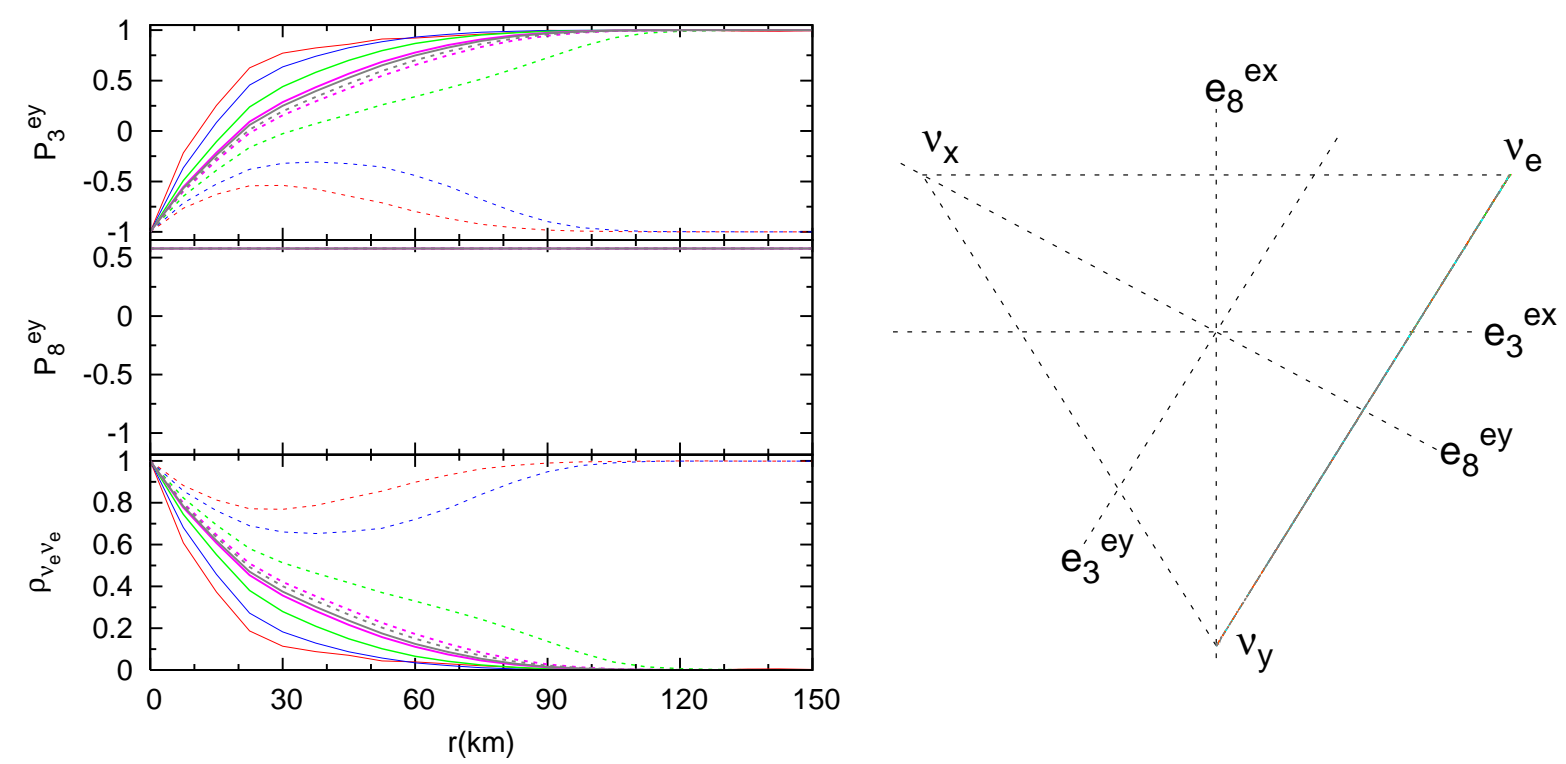

FIG. 8: Spectral splits at large $\lambda$ for neutrinos (dotted) and $33 \%$ fewer antineutrinos (undotted) with a boxspectrum of energies $E=(1-51) \mathrm{MeV}$. In the $\rho_{\nu_{e} \nu_{e}}$ plot, the energy of neutrinos (antineutrinos) increases (decreases) top downwards. The energies (in MeV) of the modes, shown in the figure, are 1.0 (Red), 1.5 (Blue), 3.5 (Green), 12.5 (Pink) and 32.0 (Grey). We take inverted hierarchy, $|\epsilon|=1 / 30, \theta_{13}=0.01$, $\mu=10^{5}(50 / r(\mathrm{~km}))^{4} \mathrm{~km}^{-1}$ and $\lambda=10 \mathrm{~km}^{-1}$. In the $\mathbf{e}_{3}-\mathbf{e}_{8}$ triangle, the evolution is always along the $\nu_{e}-\nu_{y}$ edge.

components.

For illustration, we choose two situations, with large and small $\lambda$ (Fig. 8 and 9 respectively) and the hierarchy is taken to be inverted. We choose the box-spectrum for $\nu_{e}$ and $\bar{\nu}_{e}$ energies and the flux asymmetry $\alpha=0.33$. We observe the following from the figures:

- For large $\lambda$, there is only a single split for neutrinos, which can be seen in $\mathrm{P}_{3}^{e y}$. The split is not visible in the triangle since the neutrinos are confined to the $\nu_{e}-\nu_{y}$ edge. However, the low energy neutrinos move towards $\nu_{e}$ and the high energy ones towards $\nu_{y}$.

- for small $\lambda$, the split is not only in $\mathrm{P}_{3}^{e y}$ but also in $\mathrm{P}_{8}^{e y}$. There also are oscillations with large amplitudes. Some neutrino states drift towards and ultimately reach $\nu_{y}$, while the others keep oscillating between $\nu_{e}$ and $\nu_{x}$.

The above observations can be understood as follows. For large $\lambda$, the solar mixing angle is suppressed and hence the problem reduces essentially to a two-flavor one in the $e-y$ subspace. Thus, the split is only in $\mathrm{P}_{3}^{e y}$. The split happens in neutrinos since there are more neutrinos than antineutrinos at any given energy. For small $\lambda$, in addition to the above split, there are large 

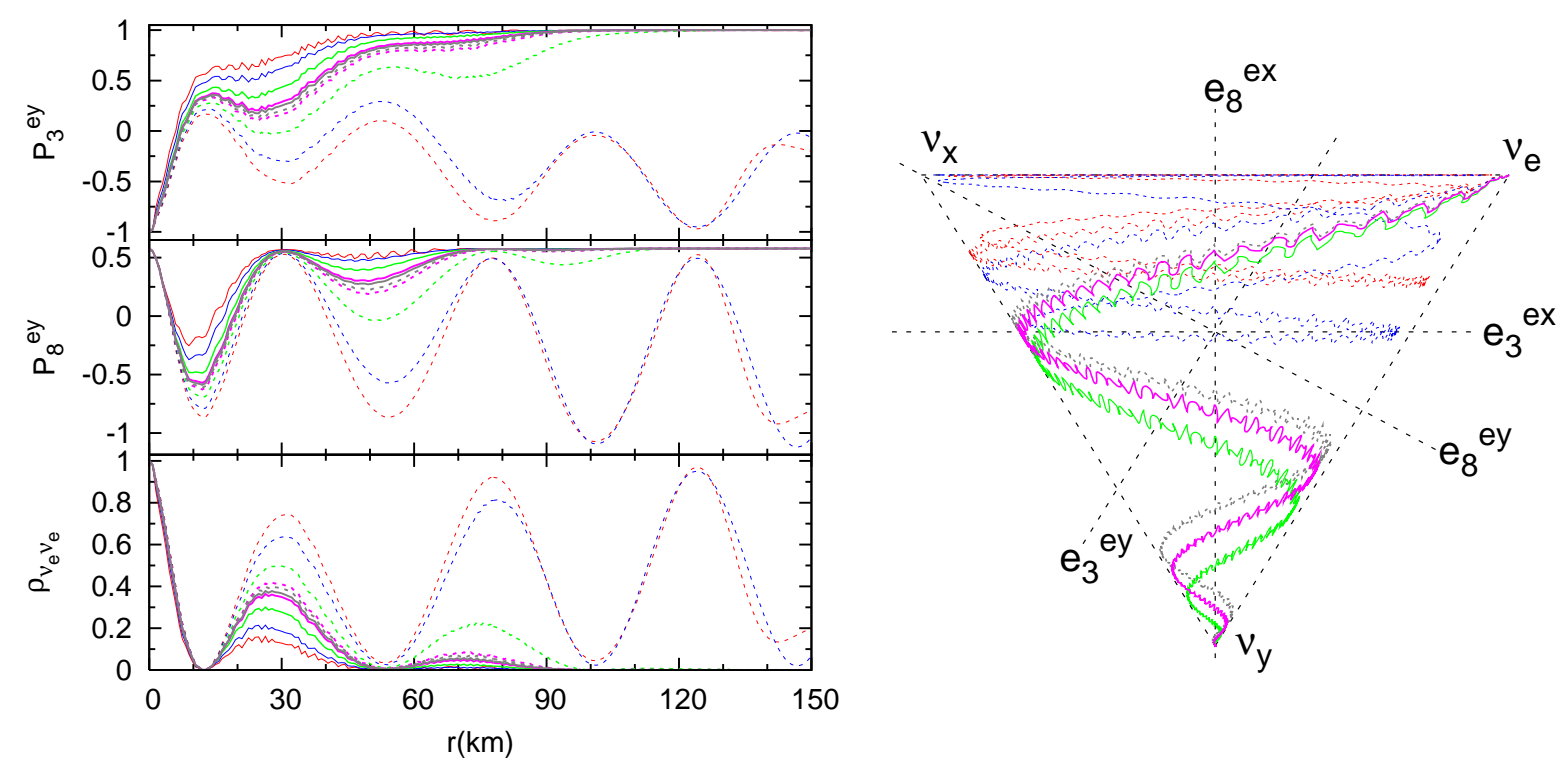

FIG. 9: Spectral splits at small $\lambda$ for neutrinos (dotted) and $33 \%$ fewer antineutrinos (undotted) with a box-spectrum of energies $(E=1-51) \mathrm{MeV}$. The conventions for lines is the same as that in Fig. 8, We take inverted hierarchy, $\epsilon=1 / 30, \theta_{13}=0.01 \mu=10^{5}(50 / r(\mathrm{~km}))^{4} \mathrm{~km}^{-1}$ and $\lambda=0.1 \mathrm{~km}^{-1}$. In the $\mathbf{e}_{3}-\mathbf{e}_{8}$ triangle, we show only some of the representative energies that have different behaviours.

$\nu_{e} \leftrightarrow \nu_{x}$ oscillations, which give rise to a split that is observable also in $\mathrm{P}_{8}^{e y}$, which was absent for large $\lambda$.

A detailed understanding of the spectral splits in the three-flavor case, including predictions for the positions of the spectral splits has been obtained [64].

\section{COLLECTIVE EFFECTS ON NEUTRINOS IN SN}

Collective effects are likely to be important in the context of neutrinos emitted from a SN. The number density of neutrinos and antineutrinos streaming off the neutrinosphere is quite large, so $\mu$ dominates over $\lambda$ and $\omega$ upto a radius of a few ten or hundred kilometers respectively. Therefore, it is likely that one or more flavor conversion mechanisms identified in Sec. III come into play in the different regions inside the star.

In this section, we study the effect of collective oscillations and their interplay with subsequent MSW transitions inside a SN. To illustrate the nature of these effects, we numerically solve for the flavor evolution equations by taking a realistic SN density profile, including the collective effects. We present results primarily for inverted hierarchy, because collective effects are not expected to 
play a significant part for normal hierarchy ${ }^{6}$. In the numerical study, we take $\left|\Delta m_{13}^{2}\right|=2.5 \times 10^{-3}$ $\mathrm{eV},|\epsilon|=1 / 30, \theta_{12}=0.6$, and two representative values of $\theta_{13}$, viz. $\theta_{13}=0.001$ and 0.1 . We then apply the formalism developed in Sec. II to the case of neutrinos streaming from the SN neutrinosphere and identify the regimes where different flavor conversion mechanisms are at work. This allows us to explain the features in the observable neutrino and antineutrino spectra, and understand the three-flavor effects.

\section{A. SN model and parameters for numerical simulation}

The SN model is defined by the following choice for the emission geometry, initial flavor dependent spectra and fluxes, the collective potential and the matter density profile. We would like to emphasize that these choices are canonical and more importantly, the specific value of the luminosity or the spatial dependence of the collective potential does not affect results significantly. Any large initial value of $\mu$ (such that it exceeds $\omega$ ) and its slow decrease with $r$ gives almost identical results. In other words, the results are not fine-tuned. However, the results would depend on the flavor spectra and the matter density profile, as these determine the initial conditions, the collective potential and the effective mixing parameters.

\section{Emission geometry}

Neutrinos with different energies and flavors start freestreaming at different $r$, but flavor evolution does not start until much later. Thus the radius of the neutrinosphere $r_{0}$ is used only to set the initial conditions. We therefore use the "bulb-model" of neutrino emission from the SN as discussed in [41] with a nominal neutrinosphere at $r_{0}=10 \mathrm{~km}$. We assume steady-state half-isotropic emission from the neutrinosphere.

\section{Initial spectra and fluxes}

The flavor-dependent primary neutrino spectra at $r_{0}$ are parametrized as [65]

$$
F_{\nu_{\alpha}}(E)=\Phi_{\nu_{\alpha}} \frac{N\left(\xi_{\alpha}\right)}{\left\langle E_{\nu_{\alpha}}\right\rangle}\left(\frac{E}{\left\langle E_{\nu_{\alpha}}\right\rangle}\right)^{\xi_{\alpha}} \exp \left[-\left(\xi_{\alpha}+1\right) \frac{E}{\left\langle E_{\nu_{\alpha}}\right\rangle}\right] \text {, }
$$

\footnotetext{
${ }^{6}$ However, during the neutronization burst phase of an O-Ne-Mg SN, this need not be true.
} 
where $N(\xi)=(1+\xi)^{1+\xi} / \Gamma(1+\xi)$. This spectrum is normalized such that $\int_{0}^{\infty} d E_{\nu_{\alpha}} F_{\nu_{\alpha}}\left(E_{\nu_{\alpha}}\right)=\Phi_{\nu_{\alpha}}$ and has the average energy $\left\langle E_{\nu_{\alpha}}\right\rangle$. The above parametrization has the advantage that the spectra can be analytically integrated, including the effects of spectral pinching through $\xi_{\alpha}$. The number flux $\Phi_{\nu_{\alpha}}$ is given by $\Phi_{\nu_{\alpha}}=L_{\nu_{\alpha}} /\left\langle E_{\nu_{\alpha}}\right\rangle$, where $L_{\nu_{\alpha}}$ is the luminosity in the flavor $\nu_{\alpha}$. We remark that an equipartition in the luminosity is often assumed for simplicity. For illustration, we choose the above parameters as

$$
\begin{aligned}
L_{\nu_{\alpha}} & =1.5 \times 10^{51} \mathrm{ergs} / \mathrm{sec}, \quad \xi_{\alpha}=3 \\
\left\langle E_{\nu_{e}}\right\rangle & =10 \mathrm{MeV}, \quad\left\langle E_{\bar{\nu}_{e}}\right\rangle=15 \mathrm{MeV}, \quad\left\langle E_{\nu_{x, y}, \bar{\nu}_{x, y}}\right\rangle=20 \mathrm{MeV}
\end{aligned}
$$

Remembering that $E \sim p=\left|\Delta m_{13}^{2}\right| /(2 \omega)$, we can rewrite the above information in terms of $\omega$, if desired. Combining Eq. (48) with the definitions of moments in (63), allows us to calculate the values of $\mathbf{D}\left(r_{0}\right), \mathbf{S}\left(r_{0}\right)$ and $\mathbf{D}^{(1)}\left(r_{0}\right)$ for the above spectrum as

$$
\begin{aligned}
\mathbf{D}\left(r_{0}\right) & =\frac{\left(\left\langle E_{\bar{\nu}_{e}}\right\rangle-\left\langle E_{\nu_{e}}\right\rangle\right)\left\langle E_{\nu_{x}}\right\rangle}{\left\langle E_{\nu_{e}}\right\rangle\left\langle E_{\nu_{x}}\right\rangle+\left\langle E_{\bar{\nu}_{e}}\right\rangle\left(4\left\langle E_{\nu_{e}}\right\rangle+\left\langle E_{\nu_{x}}\right\rangle\right)} \mathbf{e}_{e}=\frac{1}{11} \mathbf{e}_{e}, \\
\mathbf{S}\left(r_{0}\right) & =\frac{\left(\left\langle E_{\nu_{e}}\right\rangle+\left\langle E_{\bar{\nu}_{e}}\right\rangle\right)\left\langle E_{\nu_{x}}\right\rangle-2\left\langle E_{\nu_{e}}\right\rangle\left\langle E_{\bar{\nu}_{e}}\right\rangle}{\left(\left\langle E_{\nu_{e}}\right\rangle+\left\langle E_{\bar{\nu}_{e}}\right\rangle\right)\left\langle E_{\nu_{x}}\right\rangle+4\left\langle E_{\nu_{e}}\right\rangle\left\langle E_{\bar{\nu}_{e}}\right\rangle} \mathbf{e}_{e}=\frac{2}{11} \mathbf{e}_{e}, \\
\mathbf{D}^{(1)}\left(r_{0}\right) & =\frac{2 \Delta m_{13}^{2}}{3} \frac{1 /\left\langle E_{\nu_{e}}\right\rangle^{2}+1 /\left\langle E_{\bar{\nu}_{e}}\right\rangle^{2}-2 /\left\langle E_{\nu_{x}}\right\rangle^{2}}{1 /\left\langle E_{\nu_{e}}\right\rangle+1 /\left\langle E_{\bar{\nu}_{e}}\right\rangle+4 /\left\langle E_{\nu_{x}}\right\rangle} \mathbf{e}_{e}=0.215 \mathbf{e}_{e} \mathrm{~km}^{-1} .
\end{aligned}
$$

Using the above expressions, $\langle\omega\rangle \equiv \mathbf{D} \cdot \mathbf{D}^{(1)} /|\mathbf{D}|^{2}$ is calculated to be

$$
\langle\omega\rangle=2.37 \mathrm{~km}^{-1}
$$

which allows us to write $\left\langle\omega^{e y}\right\rangle=\langle\omega\rangle$ and $\left\langle\omega^{e x}\right\rangle=\epsilon\langle\omega\rangle$ in terms of $\langle\omega\rangle$, as per Eq. (103).

\section{Collective potential and matter density profile}

The collective potential for $r>r_{0}$ for the choice of parameters in Eq. (112) is given by

$$
\mu(r)=0.45 \times 10^{5} g(r) \mathrm{km}^{-1},
$$

where $g(r)$ is given in Eq. (28). For illustration, we choose the shock-wave simulation inspired density profile that corresponds to ${ }^{7}$

$$
\lambda(r)=1.84 \times 10^{6} / r^{2.4} \mathrm{~km}^{-1}
$$

The profiles of $\lambda(r)$ and $\mu(r)$ are shown in Fig. 10.

\footnotetext{
${ }^{7}$ This is the same as the one used in [13] at $t=4$ sec.
} 


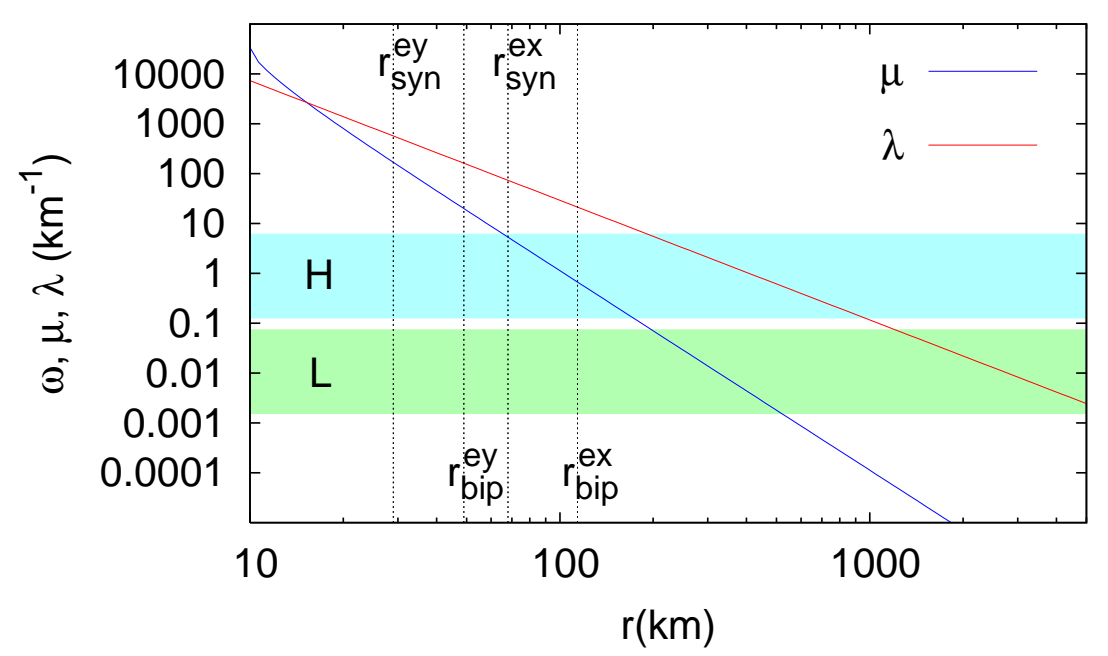

FIG. 10: The profiles of $\lambda(r)$ and $\mu(r)$ for the SN model chosen in this section, along with the bands for the MSW resonances $H$ and $L$. Also indicated are the terminal values of $r$ where synchronized /bipolar oscillations for the $e-y$ and $e-x$ flavors take place.

\section{B. Flavor conversions inside a supernova}

In this section we solve for the evolution of the neutrino density matrix numerically for the chosen density profile, and show the neutrino flavor conversions. We expect synchronized oscillations in the region where $\mu>4\left\langle\omega^{e y}\right\rangle \mathrm{S}_{3}^{e y} /\left(\mathrm{D}_{3}^{e y}\right)^{2} \approx 208 \mathrm{~km}^{-1}$ [48], which corresponds to $r_{\text {syn }}^{e y} \approx 30 \mathrm{~km}$ in our example. In the further region till $\mu \approx\left\langle\omega^{e y}\right\rangle / \mathrm{D}_{3}^{e y} \approx 26 \mathrm{~km}^{-1}$ [48], which corresponds to $r_{\text {bip }}^{e y} \approx 49 \mathrm{~km}, \nu_{e} \leftrightarrow \nu_{y}$ bipolar oscillations are expected. Beyond this region the spectral split in the $e-y$ sector should develop, and subsequently MSW resonances should take place. Similarly we calculate for the $e-x$ flavors, the relevant values of $r_{s y n}^{e x} \sim 68 \mathrm{~km}$ and $r_{b i p}^{e x} \sim 114 \mathrm{~km}$ for approximate boundaries of synchronized and bipolar oscillations in the $e-x$ sector. However, no bipolar oscillations take place in the $e-x$ sector since the corresponding hierarchy is normal. In Fig. 10, we show the positions corresponding to $r_{s y n}^{e y}, r_{b i p}^{e y}, r_{s y n}^{e x}$ and $r_{b i p}^{e x}$.

\section{Small $\theta_{13}$}

Fig. 11 shows the flavor evolutions in terms of $\mathrm{P}_{3}^{e y}, \mathrm{P}_{8}^{e y}$ and the $\mathbf{e}_{3}-\mathbf{e}_{8}$ triangle for neutrinos as well as antineutrinos, for $\theta_{13}=0.001$. This small value of $\theta_{13}$ ensures that the MSW resonance 

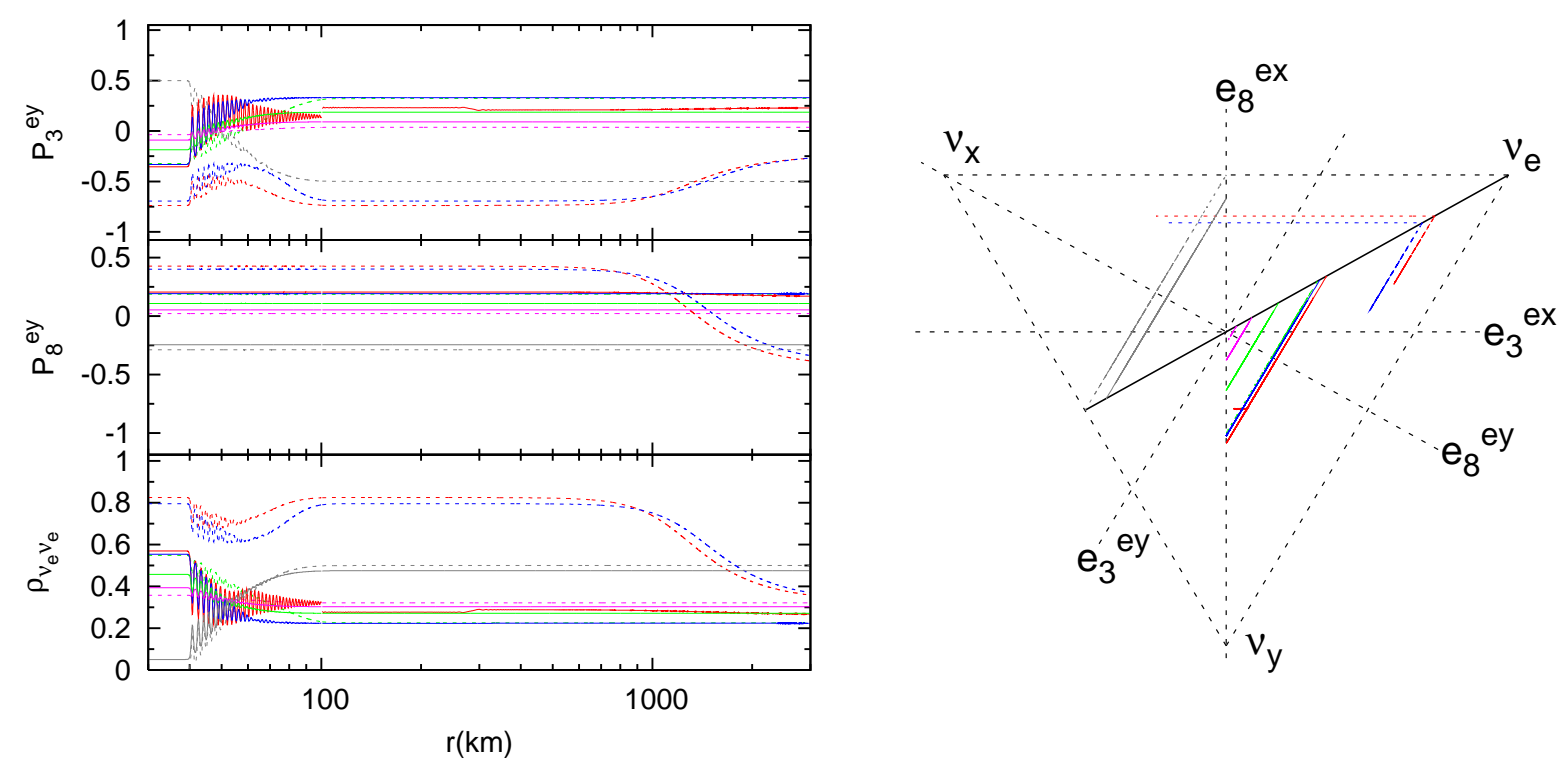

FIG. 11: The flavor evolution of representative energy modes of $\nu_{e}$ (dotted) and $\bar{\nu}_{e}$ (undotted) for the density profile in Fig.10, with $\theta_{13}=0.001$. In the $\rho_{\nu_{e} \nu_{e}}$ plot, the energy of neutrinos as well as antineutrinos increases top downwards. The energies (in $\mathrm{MeV}$ ) of the modes, shown in the figure, are 2.5 (Red), 3.6 (Blue), 9.4 (Green), 13.3 (Pink) and 50.0 (Grey). In the triangle plot, the bold line passing through $\nu_{e}$ is where all the neutrino and antineutrino states initially lie.

$H$ in antineutrinos is nonadiabatic, so that the effects of this resonance are not felt. One can then cleanly identify the collective effects. We make the following observations and interpretations based on the figure:

- All the neutrinos and antineutrinos initially lie on a line passing through $\nu_{e}$ in the $\mathbf{e}_{3}-\mathbf{e}_{8}$ triangle. This is because the initial conditions are taken to be symmetric in $\nu_{x}$ and $\nu_{y}$.

- The flavor evolution starts only at $r=40 \mathrm{~km}$, which is slightly beyond $r_{\text {synn }}^{e y}$. Before this point, the oscillations are synchronized, with a vanishing amplitude since $\lambda \gg\left\langle\omega^{e y}\right\rangle$.

- Between $r=40$ and $60 \mathrm{~km}, \nu_{e} \leftrightarrow \nu_{y}$ bipolar oscillations are observed as rapid dips in $\mathrm{P}_{3}^{e y}$, and consequently in $\rho_{\nu_{e} \nu_{e}}$. These oscillations vanish when $r \gtrsim r_{b i p}^{e y}$.

- Around $r \approx 60 \mathrm{~km}$, a spectral split develops in neutrinos along $\mathrm{P}_{3}^{e y}$. The spectral split tends to keep the low energy neutrinos at their original position, while taking the high energy neutrinos as well as almost all antineutrinos towards $\mathrm{P}_{3}^{e x}=0 .{ }^{8}$

8 There seems to be a spectral split in antineutrinos as well, at very low energies. This is similar to the observation in [48], and may be the effect of nonadiabaticity in the splitting. 

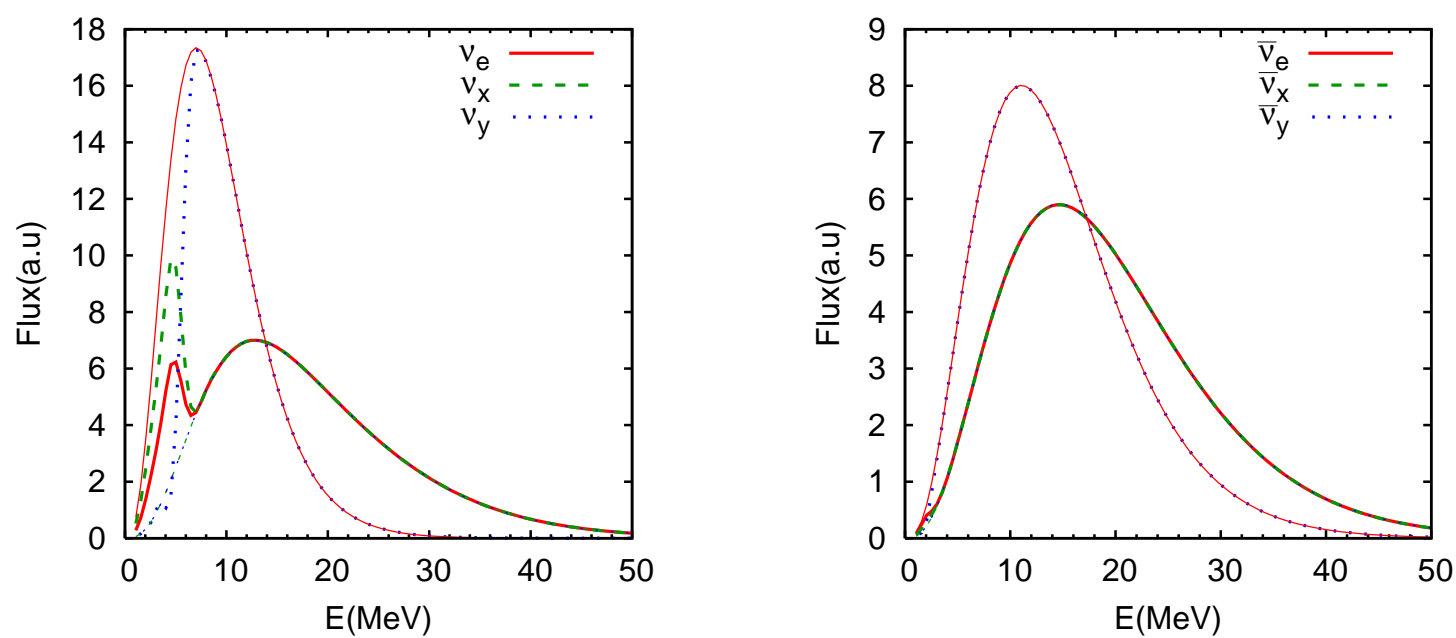

FIG. 12: Neutrino and antineutrino spectra at $r=5000 \mathrm{~km}$ for $\theta_{13}=0.001$. The $e, x$ and $y$ flavors are shown in red(solid), green(dashes) and blue(dots). The thin lines/dashes/dots are for initial spectra and thick ones for the final spectra. The $\nu_{e}$ and $\nu_{y}$ spectra get swapped for $E \gtrsim 7 \mathrm{MeV}$, whereas the lower energy $\nu_{e}$ spectrum partially mixes with $\nu_{x}$. In the antineutrino sector, the $\bar{\nu}_{e}$ and $\bar{\nu}_{y}$ spectra are almost completely swapped, while the $\bar{\nu}_{x}$ spectrum remains unaffected.

- Between $r \approx 100-1000 \mathrm{~km}$, antineutrinos of different energies undergo the $H$ resonance. However the resonance is highly nonadiabatic and does not cause any flavor conversion.

- At $r=1000 \mathrm{~km}$ and beyond, the effects of the MSW resonance $L$ come into play, resulting in $\nu_{e} \leftrightarrow \nu_{x}$ conversion. Since the high energy neutrinos are already close to $\mathrm{P}_{3}^{e x}=0$, there is effectively no flavor conversion. However the low energy neutrinos tend to convert to $\nu_{x}$, which is seen as a movement parallel to the $\nu_{e}-\nu_{x}$ edge in the $\mathbf{e}_{3}-\mathbf{e}_{8}$ triangle.

- Since all the flavor conversions can be understood as a net effect of two-flavor phenomena taking place in well-separated regions in the star, the flavor transitions in the $\mathbf{e}_{3}-\mathbf{e}_{8}$ triangle are always parallel to the $\nu_{e}-\nu_{x}$ edge or $\nu_{e}-\nu_{y}$ edge.

Thus, for a small $\theta_{13}$, the collective effects can be clearly identified, whereas the effects due to the $H$ resonance are absent. We calculate the flavor evolution till $r=5000 \mathrm{~km}$. The collective effects have almost vanished by this time. Further MSW resonances due to the shock wave [11-17], as well as possible effects of stochastic density fluctuations or turbulence [18 20] will govern flavor conversions here onwards. Our calculations thus provide initial conditions for neutrino spectra at this point.

In Fig. 12, we show the neutrino and antineutrino spectra at $r=5000 \mathrm{~km}$. We see that $\nu_{e}$ with $E \gtrsim 7 \mathrm{MeV}$ convert almost completely to $\nu_{y}$ due to the spectral split, whereas lower energy 

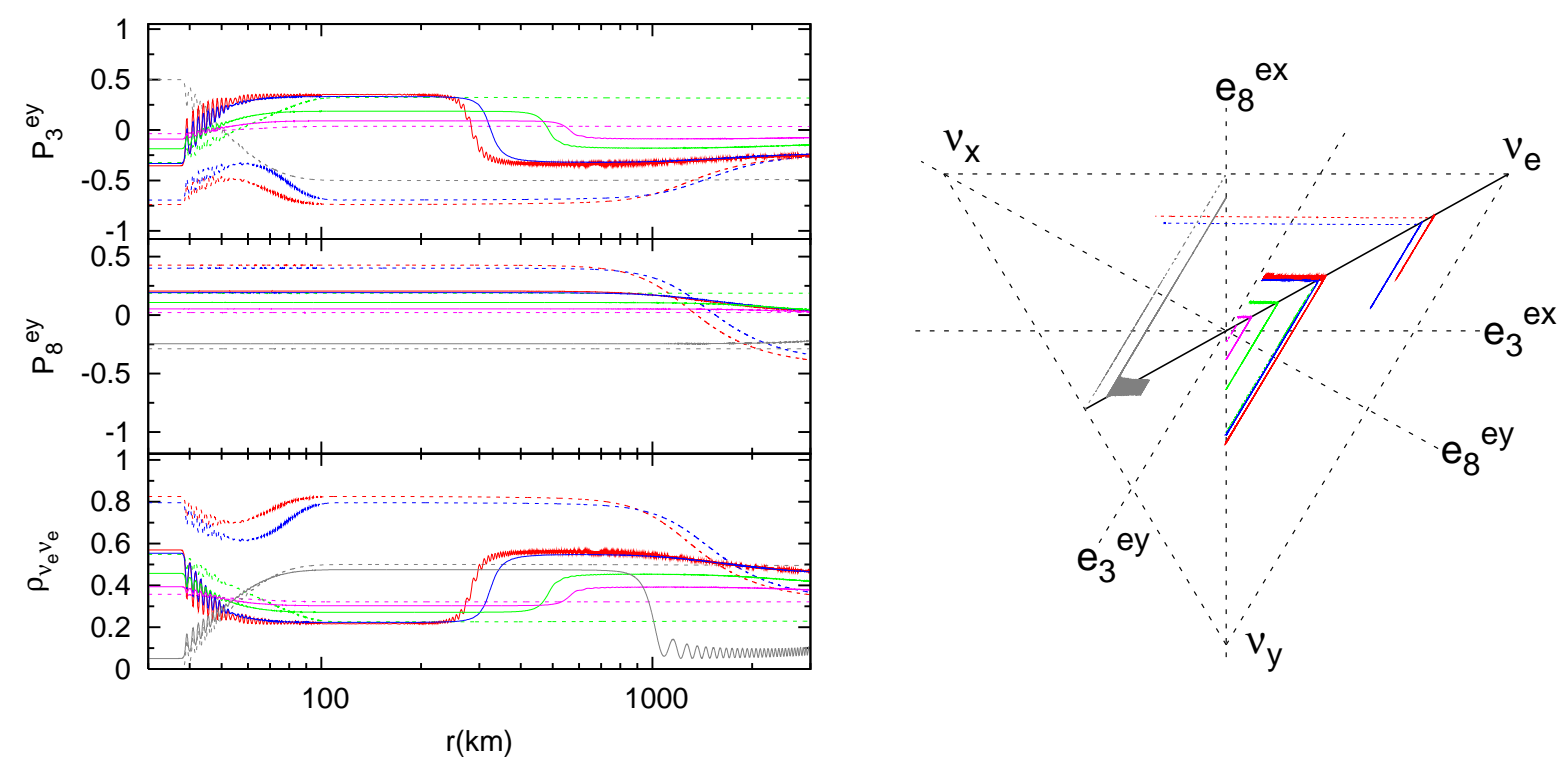

FIG. 13: The flavor evolution of same representative energy modes of $\nu_{e}\left(\right.$ dotted) and $\bar{\nu}_{e}$ (undotted) for the density profile in Fig. 10, with $\theta_{13}=0.1$. The convention for the lines is the same as in Fig. [11,

$\nu_{e}$ convert partially to $\nu_{x}$ at the $L$ resonance. In the antineutrino sector, the $\bar{\nu}_{e}$ and $\bar{\nu}_{y}$ spectra are almost all completely swapped due to the spectral split, while the $\bar{\nu}_{x}$ spectrum remains unaffected.

\section{Large $\theta_{13}$}

At large $\theta_{13}$ values, the $H$ resonance at $r \approx 100-1000 \mathrm{~km}$ is adiabatic, and causes significant flavor conversions in antineutrinos. In Fig. 13, we show the flavor evolution for $\theta_{13}=0.1$. While the signatures of synchronized and bipolar oscillations as well as the spectral split remain identical to the $\theta_{13}=0.001$ case, the $H$ resonance can be seen to change the antineutrino picture substantially. The conversions in the neutrino sector, on the other hand, are identical to the small $\theta_{13}$ case. The following observations can be made from the figure.

- The spectral split gives rise to a complete $\bar{\nu}_{e}-\bar{\nu}_{y}$ conversion, which takes antineutrinos to $\mathrm{P}_{3}^{e x}=0$.

- The $H$ resonance again swaps the $\bar{\nu}_{e}-\bar{\nu}_{y}$ spectra, thus undoing the earlier effect of the spectral split. This takes the antineutrinos back to their starting position in the triangle.

- Antineutrinos are now not on the $\mathrm{P}_{3}^{e x}=0$ line as in the small $\theta_{13}$ case. As a result, the large value of $\theta_{12}$ causes substantial $\bar{\nu}_{e}-\bar{\nu}_{x}$ conversion as the neutrinos emerge from the $L$ resonance region. 

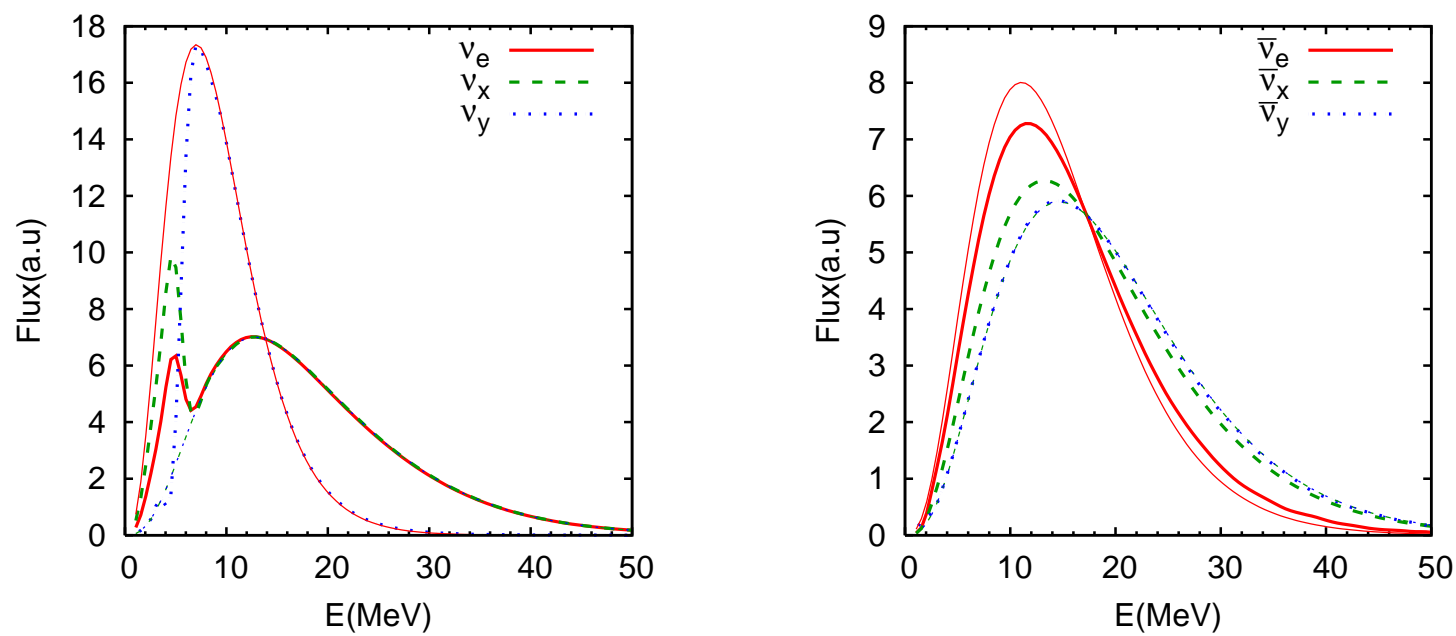

FIG. 14: Neutrino and antineutrino spectra at $r=5000 \mathrm{~km}$ for $\theta_{13}=0.1$. The $e, x$ and $y$ flavors are shown in red(solid), green(dashes) and blue(dots). The thin lines/dashes/dots are for initial spectra and thick ones for the final spectra. The $\nu_{e}$ and $\nu_{y}$ spectra get swapped for $E \gtrsim 7 \mathrm{MeV}$, whereas the lower energy $\nu_{e}$ spectrum partially mixes with $\nu_{x}$. In the antineutrino sector, the $\bar{\nu}_{e}$ and $\bar{\nu}_{x}$ spectra are partially mixed, while the $\bar{\nu}_{y}$ spectrum remains unaffected.

The neutrino and antineutrino spectra at $r=5000 \mathrm{~km}$ are shown in Fig. 14. We see that the neutrino spectra have the same characteristics as for small $\theta_{13}$. In the antineutrino sector, complete $\bar{\nu}_{e}-\bar{\nu}_{y}$ spectral split and the reconversion at the $H$ resonance cancel each other, whereas the large value of $\theta_{12}$ partially mixes the $\bar{\nu}_{e}-\bar{\nu}_{x}$ spectra.

The value of $\theta_{13}$ thus affects the $\bar{\nu}_{e}$ spectra substantially. At larger $\theta_{13}$ values, where the $H$ resonance is more adiabatic, the $\bar{\nu}_{e}$ spectrum is softer. The $\bar{\nu}_{x}$ spectrum is also affected at large $\theta_{13}$, as opposed to the small $\theta_{13}$ case.

\section{Summarized results}

It is thus clear that the neutrino fluxes that reach Earth from a $\mathrm{SN}$, are very different from the primary fluxes. In particular for inverted hierarchy, we learn that the $\nu_{e}$ and $\nu_{y}$ spectra are exchanged above a certain split-energy $E_{c}$ due to collective effects. For antineutrinos the swap occurs over the complete spectrum. In the normal hierarchy, collective effects do not have a significant effect. The MSW conversions cause further flavor conversions, and while the conversion probabilities have not changed from the traditional expectation, the primary fluxes entering the resonances are now vastly different. This leads to different flavor composition of the fluxes of neutrinos and antineutrinos arriving on Earth, than was traditionally expected. These fluxes can 
be calculated using our understanding of collective effects and the level-crossing diagrams. At the detectors on Earth one is typically sensitive to the $\nu_{e}$ and/or $\bar{\nu}_{e}$ flux, and so we summarize the expectations for these fluxes in Table I. The expressions in the table are able to describe all the features of $\nu_{e}$ and $\bar{\nu}_{e}$ spectra in Figs. 12 and 14,

\begin{tabular}{ll}
\hline Normal hierarchy & Inverted hierarchy \\
\hline$F_{\nu_{e}}^{o b s}=s_{12}^{2}\left(P_{H} F_{\nu_{e}}+\left(1-P_{H}\right) F_{\nu_{y}}\right)+c_{12}^{2} F_{\nu_{x}}$ & $F_{\nu_{e}}^{o b s}= \begin{cases}s_{12}^{2} F_{\nu_{e}}+c_{12}^{2} F_{\nu_{x}} & \left(E<E_{c}\right) \\
s_{12}^{2} F_{\nu_{y}}+c_{12}^{2} F_{\nu_{x}} & \left(E>E_{c}\right)\end{cases}$ \\
$F_{\bar{\nu}_{e}}^{o b s}=c_{12}^{2} F_{\bar{\nu}_{e}}+s_{12}^{2} F_{\bar{\nu}_{x}}$ & $F_{\bar{\nu}_{e}}^{o b s}=s_{12}^{2} F_{\bar{\nu}_{x}}+c_{12}^{2}\left(\left(1-P_{H}\right) F_{\bar{\nu}_{e}}+P_{H} F_{\bar{\nu}_{y}}\right)$ \\
\hline
\end{tabular}

TABLE I: Neutrino and antineutrino fluxes arriving on Earth from a SN.

We have taken the $L$ resonance to be adiabatic. In the case of multiple $H$ resonances, as may occur during the shock wave propagation or turbulence, $P_{H}$ may be taken to be the effective jump probability (it may have a nontrivial dependence on energy and time). Note that Earth matter effects are present only when $F_{\nu_{e} / \bar{\nu}_{e}}^{o b s}$ is a nontrivial combination of $F_{\nu_{e} / \bar{\nu}_{e}}$ and $F_{\nu_{x}, \nu_{y} / \bar{\nu}_{x}, \bar{\nu}_{y}}$.

\section{CONCLUSIONS}

We have developed a formalism to analyze neutrino flavor conversion effects in the full threeflavor framework. It employs the Bloch vector representation for $3 \times 3$ density matrices, and naturally generalizes the spin-precession analogy to three flavors. In particular, it is capable of describing three-flavor collective neutrino conversion effects inside a core collapse supernova, like synchronized oscillations, bipolar oscillations and spectral split, which have till now been analytically studied mostly in the two-flavor limit.

We explicitly extend the earlier two-flavor analysis of neutrino flavor conversions inside the SN, which includes neutrino-neutrino interactions, to three flavors, where we neglect the CP violation in the neutrino sector. We use the modified flavor basis $\left(\nu_{e}, \nu_{x}, \nu_{y}\right)$, which is rotated from the flavor basis $\left(\nu_{e}, \nu_{\mu}, \nu_{\tau}\right)$ so as to get rid of the mixing angle $\theta_{23}$. We also work in the steady state approximation so that there is no explicit time dependence in the density matrix, assume spherical symmetry and half-isotropic neutrino source, and employ the single-angle approximation that has been shown to be valid in the two-flavor case. This leads to the equations of a gyroscope in eight dimensions, similar to the three dimensional gyrosope equations in the two-flavor case.

In the three-flavor formalism, the density matrix is represented by an eight-dimensional Bloch vector $\mathbf{P}$. However, the flavor content is determined only by the two components $\mathrm{P}_{3}$ and $\mathrm{P}_{8}$ 
of $\mathbf{P}$ after evolution. Motivated by this observation, we propose the " $\mathbf{e}_{3}-\mathbf{e}_{8}$ " triangle diagram to represent the flavor content of any neutrino state by the projection of $\mathbf{P}$ on the $\mathbf{e}_{3}-\mathbf{e}_{8}$ plane, which we have termed $\mathrm{P}$. This not only allows us to visualize the three-flavor transformations in a convenient way, but also allows us to quantify the extent of three-flavor effects over and above the two-flavor results.

A "heavy-light" factorization holds in the three-flavor treatment for certain initial conditions, so that the three-flavor results may be understood as the two-flavor results with $\Delta m_{\text {atm }}^{2}$ modified with terms that depend on $\Delta m_{\odot}^{2}$. Indeed, in certain situations, the three-flavor neutrino conversions may be factorized into three two-flavor oscillations with hierarchical frequencies. In such cases, the three-flavor conversion probabilities may be constructed from two-flavor results by considering the modulation of higher frequency modes by lower frequency modes.

We have compared our analytic results with the numerical ones for simple cases of an initial pure $\nu_{e}$ state, constant matter densities and no collective effects, as well as for synchronized oscillations, and have found a good agreement even when we ignore the modulation due to the lowest frequency. The additional effect of the third neutrino in these cases is limited to the excursions of the orbit of P towards $\nu_{x}$, and has already been well studied (though without the Bloch vector treatment). In the absence of collective effects, though the evolution of all energies is different, the orbit of $\mathrm{P}$ can be seen to be an energy-independent quantity. In the synchronized case, neutrinos of all energies are seen to oscillate with a common frequency, and even undergo MSW resonances at the same matter density and with the same adiabaticity.

In the case of bipolar oscillations, the addition of the third neutrino changes the situation significantly. The analytical results are not so easy to obtain, however the numerical results for an inverted hierarchy show a "petal" pattern in the $\mathbf{e}_{3}-\mathbf{e}_{8}$ triangle, which can be explained by the combination of $\nu_{e} \leftrightarrow \nu_{y}$ bipolar oscillations and $\nu_{e} \leftrightarrow \nu_{x}$ sinusoidal oscillations. The value of the MSW potential also plays an important role in determining the extent of the effect of the third flavor. This needs to be explored in more detail.

The spectral split occurs in neutrinos in the inverted hierarchy when one starts with $\nu_{e}$, owing to the unstable position of the eight-dimensional gyroscope in this case. The $\nu_{e}$ above a certain energy, and almost all $\bar{\nu}_{e}$, completely convert to $\nu_{y}$ and $\bar{\nu}_{y}$ respectively. There are no additional split effects from the introduction of the third flavor since the hierarchy in the solar sector is normal. This, however, could change if neutrinos are not in a pure $\nu_{e}$ state as they enter the bipolar region.

We have simulated the neutrino flavor conversions numerically by taking a realistic density profile for the SN, and have shown the flavor conversions for inverted hierarchy and two $\theta_{13}$ values 
in the cooling phase. In such a scenario, it is easily possible to identify regions where different collective as well as MSW effects dominate. We are able to predict the regions in which these effects take place, and our three-flavor formalism can explain the features of flavor conversions therein. We also point out an interplay between the collective and MSW effects. For example, the $H$ resonance cancels the effect of the spectral split for antineutrinos, whereas the spectral split makes the $L$ resonance irrelevant for neutrinos above the split energy. If the hierarchy were normal, the collective effects would be effectively absent in the cooling phase.

In conclusion, a complete understanding of the neutrino flavor conversions inside a SN requires a three-flavor treatment. In this paper, we have developed a formalism to handle this analytically, provided a method to estimate the three-flavor probabilities using the two-flavor results in certain situations, and have pointed out an interplay between collective and MSW effects, which can be easily understood with the formalism.

\section{Acknowledgements}

We would like to thank K. Damle, S. Gupta, R. Loganayagam, A. Mirizzi, G. G. Raffelt for useful discussions and insightful comments, and V. Tripathi for factors of three. This work is partly supported through the Partner Group program between the Max Planck Institute for Physics and Tata Institute of Fundamental Research.

\section{Appendix A: Notation}

This paper uses 3 -vectors, $3 \times 3$ matrices, 8 -vectors, their components and their projections in two-dimensional planes. We have tried to be consistent in the use of fonts for all these objects. The convention followed for indices is:

- $i, j, k \in\{1,2,3\}$ index mass eigenstates

- $\alpha, \beta \in\{e, x, y\}$ index modified flavor eigenstates

- $a, b, c \in\{1,2,3,4,5,6,7,8\}$ are $\mathrm{SU}(3)$ indices.

The convention followed for symbols is:

- Three-dimensional vectors are denoted by smallcase boldfaced letters, e.g. p, q, r, v. 
- $3 \times 3$ matrices are denoted in the regular math mode, e.g. $\rho, H, I, M, O, R_{i j}, U, V, \Lambda_{a}$. Note however the exceptions, energy $E$ and the Fermi constant $G_{F}$, that are also respresented in this font.

- Sets of $3 \times 3$ matrices are denoted by blackboard-bold font, e.g. $\mathbb{K}, \mathbb{Q}$.

- Eight-dimensional vectors are written in bold capital letters, e.g. B, D, P, S, X. Exceptions are $\mathbf{e}_{a}$, which are vectors along the coordinate axes, and therefore conventionally written in smallcase.

- The components of an eight-vector $\mathbf{X}$ are written as $\mathbf{X}_{a}$.

- $\boldsymbol{\Lambda}$ is a eight-dimensional vector whose components are $3 \times 3$ matrices $\Lambda_{a}$.

- Projections of eight-vectors on the $\mathbf{e}_{3}-\mathbf{e}_{8}$ plane, e.g. P, as well as the rotation and evolution matrices on that plane, e.g. R, S are written in sans-serif font.

[1] G. G. Raffelt, "Supernova neutrino observations: What can we learn?," astro-ph/0701677.

[2] A. Dighe, "Neutrinos from a core collapse supernova," AIP Conf. Proc. 981 (2008) 75 arXiv:0712.4386 [hep-ph].

[3] L. Wolfenstein, "Neutrino Oscillations In Matter," Phys. Rev. D 17, 2369 (1978).

[4] S. P. Mikheev and A. Yu. Smirnov, "Resonance Enhancement Of Oscillations In Matter And Solar Neutrino Spectroscopy," Yad. Fiz. 42, 1441 (1985) [Sov. J. Nucl. Phys. 42, 913 (1985)].

[5] G. M. Fuller, R. W. Mayle, J. R. Wilson, and D. N. Schramm "Resonant neutrino oscillations and stellar collapse," Astrophys. J. 322795 (1987).

[6] A. S. Dighe and A. Y. Smirnov, "Identifying the neutrino mass spectrum from the neutrino burst from a supernova," Phys. Rev. D 62 (2000) 033007 hep-ph/9907423.

[7] C. Lunardini and A. Yu. Smirnov, "Probing the neutrino mass hierarchy and the 13-mixing with supernovae," JCAP 0306, 009 (2003) hep-ph/0302033.

[8] A. S. Dighe, M. T. Keil and G. G. Raffelt, "Detecting the neutrino mass hierarchy with a supernova at IceCube," JCAP 0306, 005 (2003) hep-ph/0303210.

[9] A. S. Dighe, M. T. Keil and G. G. Raffelt, "Identifying earth matter effects on supernova neutrinos at a single detector," JCAP 0306, 006 (2003) hep-ph/0304150.

[10] A. S. Dighe, M. Kachelriess, G. G. Raffelt and R. Tomas, "Signatures of supernova neutrino oscillations in the earth mantle and core," JCAP 0401, 004 (2004) hep-ph/0311172. 
[11] R. C. Schirato and G. M. Fuller, "Connection between supernova shocks, flavor transformation, and the neutrino signal," astro-ph/0205390.

[12] K. Takahashi, K. Sato, H. E. Dalhed and J. R. Wilson, "Shock propagation and neutrino oscillation in supernova," Astropart. Phys. 20, 189 (2003) astro-ph/0212195.

[13] G. L. Fogli, E. Lisi, A. Mirizzi, and D. Montanino, "Analysis of energy- and time-dependence of supernova shock effects on neutrino crossing probabilities," Phys. Rev. D 68, 033005 (2003) hep-ph/0304056.

[14] R. Tomàs, M. Kachelrieß, G. Raffelt, A. Dighe, H. T. Janka and L. Scheck, "Neutrino signatures of supernova shock and reverse shock propagation," JCAP 0409, 015 (2004) astro-ph/0407132.

[15] G. L. Fogli, E. Lisi, A. Mirizzi and D. Montanino, "Probing supernova shock waves and neutrino flavor transitions in next-generation water-Cherenkov detectors," JCAP 0504, 002 (2005) hep-ph/0412046.

[16] V. Barger, P. Huber and D. Marfatia, "Supernova neutrinos can tell us the neutrino mass hierarchy independently of flux models," Phys. Lett. B 617, 167 (2005) hep-ph/0501184.

[17] B. Dasgupta and A. Dighe, "Phase effects in neutrino conversions during a supernova shock wave," Phys. Rev. D 75 (2007) 093002 hep-ph/0510219.

[18] G. L. Fogli, E. Lisi, A. Mirizzi and D. Montanino, "Damping of supernova neutrino transitions in stochastic shock-wave density profiles," JCAP 0606, 012 (2006) hep-ph/0603033.

[19] S. Choubey, N. P. Harries and G. G. Ross, "Probing neutrino oscillations from supernovae shock waves via the IceCube detector," Phys. Rev. D 74, 053010 (2006) hep-ph/0605255.

[20] A. Friedland and A. Gruzinov, "Neutrino signatures of supernova turbulence," astro-ph/0607244.

[21] J. P. Kneller, G. C. McLaughlin and J. Brockman, "Oscillation Effects and Time Variation of the Supernova Neutrino Signal," arXiv:0705.3835 [astro-ph].

[22] J. T. Pantaleone, "Dirac neutrinos in dense matter," Phys. Rev. D 46510 (1992).

[23] J. Pantaleone, "Neutrino oscillations at high densities," Phys. Lett. B 287, 128 (1992).

[24] M. J. Thomson and B. H. J. McKellar, "The nonlinear MSW equation," Phys. Lett. B 259, 113 (1991).

[25] G. Sigl and G. Raffelt, "General kinetic description of relativistic mixed neutrinos," Nucl. Phys. B 406, 423 (1993).

[26] B. H. J. McKellar and M. J. Thomson, "Oscillating doublet neutrinos in the early universe," Phys. Rev. D 49, 2710 (1994).

[27] S. Samuel, "Neutrino oscillations in dense neutrino gases," Phys. Rev. D 48, 1462 (1993).

[28] V. A. Kostelecký and S. Samuel, "Self-maintained coherent oscillations in dense neutrino gases," Phys. Rev. D 52, 621 (1995) hep-ph/9506262.

[29] J. Pantaleone, "Stability of incoherence in an isotropic gas of oscillating neutrinos," Phys. Rev. D 58, 073002 (1998).

[30] S. Samuel, "Bimodal coherence in dense selfinteracting neutrino gases," Phys. Rev. D 53, 5382 (1996) hep-ph/9604341.

[31] S. Pastor, G. G. Raffelt and D. V. Semikoz, "Physics of synchronized neutrino oscillations caused by self-interactions," Phys. Rev. D 65, 053011 (2002) hep-ph/0109035. 
[32] S. Hannestad, G. G. Raffelt, G. Sigl and Y. Y. Y. Wong, "Self-induced conversion in dense neutrino gases: Pendulum in flavour space," Phys. Rev. D 74, 105010 (2006) [Erratum-ibid. D 76, 029901 (2007)] astro-ph/0608695.

[33] H. Duan, G. M. Fuller, J. Carlson and Y. Z. Qian, "Analysis of Collective Neutrino Flavor Transformation in Supernovae," Phys. Rev. D 75, 125005 (2007) astro-ph/0703776.

[34] G. G. Raffelt and A. Y. Smirnov, "Self-induced spectral splits in supernova neutrino fluxes," Phys. Rev. D 76081301 (2007) arXiv:0705.1830 [hep-ph].

[35] G. G. Raffelt and A. Y. Smirnov, "Adiabaticity and spectral splits in collective neutrino transformations," arXiv:0709.4641 [hep-ph].

[36] J. T. Pantaleone, "Neutrino flavor evolution near a supernova's core," Phys. Lett. B 342, 250 (1995) astro-ph/9405008.

[37] Y. Z. Qian and G. M. Fuller, "Neutrino-neutrino scattering and matter enhanced neutrino flavor transformation in Supernovae," Phys. Rev. D 51, 1479 (1995) astro-ph/9406073.

[38] G. Sigl, "Neutrino mixing constraints and supernova nucleosynthesis," Phys. Rev. D 51, 4035 (1995) astro-ph/9410094.

[39] S. Pastor and G. Raffelt, "Flavor oscillations in the supernova hot bubble region: Nonlinear effects of neutrino background," Phys. Rev. Lett. 89, 191101 (2002) astro-ph/0207281.

[40] A. B. Balantekin and H. Yüksel, "Neutrino mixing and nucleosynthesis in core-collapse supernovae," New J. Phys. 7, 51 (2005) astro-ph/0411159].

[41] H. Duan, G. M. Fuller, J. Carlson and Y. Z. Qian, "Simulation of coherent non-linear neutrino flavor transformation in the supernova environment. I: Correlated neutrino trajectories," Phys. Rev. D 74, 105014 (2006) astro-ph/0606616.

[42] H. Duan, G. M. Fuller, J. Carlson and Y. Z. Qian, "Coherent development of neutrino flavor in the supernova environment," Phys. Rev. Lett. 97, 241101 (2006) astro-ph/0608050.

[43] G. M. Fuller and Y. Z. Qian, "Simultaneous flavor transformation of neutrinos and antineutrinos with dominant potentials from neutrino neutrino forward scattering," Phys. Rev. D 73, 023004 (2006) astro-ph/0505240.

[44] H. Duan, G. M. Fuller and Y. Z. Qian, "Collective neutrino flavor transformation in supernovae," Phys. Rev. D 74, 123004 (2006) astro-ph/0511275.

[45] H. Duan, G. M. Fuller and Y. Z. Qian, "A Simple Picture for Neutrino Flavor Transformation in Supernovae," Phys. Rev. D 76, 085013 (2007) arXiv:0706.4293 [astro-ph].

[46] H. Duan, G. M. Fuller, J. Carlson and Y. Q. Zhong, "Neutrino Mass Hierarchy and Stepwise Spectral Swapping of Supernova Neutrino Flavors," arXiv:0707.0290 [astro-ph].

[47] A. Esteban-Pretel, S. Pastor, R. Tomàs, G. G. Raffelt and G. Sigl, "Decoherence in supernova neutrino transformations suppressed by deleptonization," arXiv:0706.2498 [astro-ph].

[48] G. L. Fogli, E. Lisi, A. Marrone and A. Mirizzi, "Collective neutrino flavor transitions in supernovae and the role of trajectory averaging," arXiv:0707.1998 [hep-ph]. 
[49] G. G. Raffelt and G. Sigl, "Self-induced decoherence in dense neutrino gases," Phys. Rev. D 75, 083002 (2007) hep-ph/0701182.

[50] H. Duan, G. M. Fuller, J. Carlson and Y. Z. Qian, "Flavor Evolution of the Neutronization Neutrino Burst from an O-Ne-Mg Core-Collapse Supernova," arXiv:0710.1271 [astro-ph].

[51] V. A. Kostelecký, J. Pantaleone and S. Samuel, "Neutrino oscillation in the early universe," Phys. Lett. B 315, 46 (1993).

[52] V. A. Kostelecký and S. Samuel, "Neutrino oscillations in the early universe with an inverted neutrino mass hierarchy," Phys. Lett. B 318, 127 (1993).

[53] V. A. Kostelecký and S. Samuel, "Nonlinear neutrino oscillations in the expanding universe," Phys. Rev. D 49, 1740 (1994).

[54] V. A. Kostelecký and S. Samuel, "Neutrino oscillations in the early universe with nonequilibrium neutrino distributions," Phys. Rev. D 52, 3184 (1995) hep-ph/9507427.

[55] V. A. Kostelecký and S. Samuel, "Nonequilibrium neutrino oscillations in the early universe with an inverted neutrino mass hierarchy," Phys. Lett. B 385, 159 (1996) hep-ph/9610399.

[56] A. D. Dolgov, S. H. Hansen, S. Pastor, S. T. Petcov, G. G. Raffelt and D. V. Semikoz, "Cosmological bounds on neutrino degeneracy improved by flavor oscillations," Nucl. Phys. B 632363 (2002) hep-ph/0201287.

[57] Y. Y. Y. Wong, "Analytical treatment of neutrino asymmetry equilibration from flavour oscillations in the early universe," Phys. Rev. D 66, 025015 (2002) hep-ph/0203180.

[58] Y. Y. Y. Wong, "Synchronised neutrino oscillations from self interaction and associated applications," AIP Conf. Proc. 655, 240 (2003) hep-ph/0211045.

[59] K. N. Abazajian, J. F. Beacom and N. F. Bell, "Stringent constraints on cosmological neutrino antineutrino asymmetries from synchronized flavor transformation," Phys. Rev. D 66, 013008 (2002) astro-ph/0203442.

[60] A. Esteban-Pretel, S. Pastor, R. Tomas, G. G. Raffelt and G. Sigl, "Mu-tau neutrino refraction and collective three-flavor transformations in supernovae," arXiv:0712.1137 [astro-ph].

[61] C. Y. Cardall, "Liouville equations for neutrino distribution matrices," arXiv:0712.1188 [astro-ph].

[62] G. Kimura, "The Bloch Vector for N-Level Systems," Phys. Lett. A 314, 339 (2003) quant-ph/0301152.

[63] C. W. Kim, J. Kim and W. K. Sze, "Geometrical representation of neutrino oscillations in vacuum and matter," Phys. Rev. D 37, 1072 (1988).

[64] B. Dasgupta, A. Dighe, A. Mirizzi and G. G. Raffelt, "Spectral split in prompt supernova neutrino burst: Analytic three-flavor treatment," arXiv:0801.1660 [hep-ph].

[65] M. T. Keil, G. G. Raffelt and H. T. Janka, "Monte Carlo study of supernova neutrino spectra formation," Astrophys. J. 590, 971 (2003) astro-ph/0208035. 